\title{
Constraining the mass-concentration relation through weak lensing peak function
}

\author{
R. Mainini, ${ }^{a}$ A. Romano ${ }^{b}$ \\ ${ }^{a}$ Physics Department, University of Milano-Bicocca, \\ Piazza della Scienza 3, I20126, Milano, Italy \\ ${ }^{b}$ INAF- Astronomical Observatory of Rome, \\ via Frascati 33, I00044, Monte Porzio Catone (RM), Italy \\ E-mail: roberto.mainini@mib.infn.it
}

\begin{abstract}
Halo masses and concentrations have been studied extensively, by means of $\mathrm{N}-$ body simulations as well as observationally, during the last decade. Nevertheless, the exact form of the mass-concentration relation is still widely debated. One of the most promising method to estimate masses and concentrations relies on gravitational lensing from massive halos. Here we investigate the impact of the mass-concentration relation on halo peak abundance in weak lensing shear maps relying on the aperture mass method for peak detections. After providing a prescription to take into account the concentration dispersion (always neglected in previous works) in peak number counts predictions, we assess their power to constrain the mass-concentration relation by means of Fisher matrix technique. We find that, when combined with different cosmological probes, peak statistics information from near-future weak lensing surveys provides an interesting and complementary alternative method to lessen the long standing controversy about the mass-concentration relation.
\end{abstract}




\section{Contents}

1 Introduction $\quad 1$

2 Aperture Mass 4

2.1 Filter function 5

2.2 Halo profile and mass-concentration relation 5

$\begin{array}{lll}2.3 \text { Galaxy survey } & 6\end{array}$

$\begin{array}{lll}3 & \text { Peak function } & 7\end{array}$

$\begin{array}{lll}3.1 & M_{a p} \text { probability distribution function } & 7\end{array}$

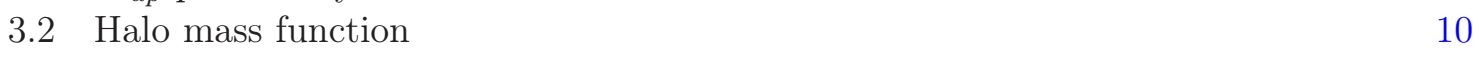

$\begin{array}{lll}3.3 & \text { Peak number counts } & 12\end{array}$

$\begin{array}{lll}4 & \text { Fisher matrix forecasts } & 17\end{array}$

5 Discussion and conclusions 23

\section{Introduction}

Weak gravitational lensing (WL) has emerged over the last decade as one of the most promising methods for testing cosmology and gravity, and unveiling the nature of Dark Energy (DE) and Dark Matter (DM). It relies on the accurate measurement of the small shape distortions of background galaxies due to the bending of light by intervening matter distribution. The smallness of these distortions requires high-quality data and a statistical approach so that the lensing shear signal needs to be measured on a large number of sources.

A number of surveys (e.g. GaBoDS [1], CFHTLS ${ }^{1}$ ) have already shown the WL capacity for constraining cosmological models through cosmic shear measurements (see e.g. [1-3]). Several other, more ambitious, surveys both ground- $\left(\mathrm{KIDS}^{2}, \mathrm{PanSTARRS}^{3}, \mathrm{DES}^{4}, \mathrm{LSST}^{5}\right.$ ) and space-based (Euclid ${ }^{6}$, WFIRST $^{7}$ ) are being performed or planned. They will map hundreds of millions of galaxy redshifts and billions of galaxy images. For instance, the Euclid mission $[4,5]$ aims to map half of the sky in imaging as well as in broad-band spectroscopy up to a redshift $z \sim 2-3$ with a median redshift of the order of unity. The information contained in these data will permit to measure the matter clustering with unprecedented accuracy.

Among the WL probes, it has been shown that the abundance of peaks in shear maps is a sensitive probe of cosmology [6]. This was tested by [7] and [8] using ray-tracing in $\mathrm{N}_{-}$ body simulations. Shear peaks are regions with high signal-to-noise ratios, snr, associated to massive halos (or galaxy clusters) or produced by the alignment of smaller mass concentrations along the line-of-sight. Their number and spatial distribution, therefore, carry information about the underlying cosmology through fundamental parameters as the total matter density

\footnotetext{
${ }^{1}$ http://www.cfht.hawaii.edu/Science/CFHLS

${ }^{2}$ http://kids.strw.leidenuniv.nl

${ }^{3}$ http://pan-starrs.ifa.hawaii.edu

${ }^{4}$ http://www.darkenergysurvey.org

${ }^{5}$ http://www.lsst.org

${ }^{6}$ http://www.euclid-ec.org

${ }^{7}$ http://wfirst.gsfc.nasa.gov
} 
of the universe $\Omega_{m}$, the normalization of the power spectrum $\sigma_{8}$, and the evolution of the DE equation of state $w(z)$.

Unlike other available techniques for detecting massive halos (e.g. optical and X-ray identification, Sunyaev-Zeldovich effect, etc), WL does not require any assumption on their dynamical or evolutionary state. Its advantage is to be only sensitive to the mass along the line-of-sight offering, in principle, the opportunity to construct mass-selected halo samples, which can be compared directly with theoretical predictions (e.g. N-body simulations) without assuming any mass-observable relation. Nevertheless, that WL provides truly massselected halo samples it is not strictly true. Large scale structures (LSS), into which massive halos are embedded, contribute to the lensing signal. Usually, these projection effects add noise to the halo signal or can result in false positive peaks in the shear map [9-11]. Further, intrinsic ellipticities of galaxies introduce an irremovable noise. All these sources of noise then compromise purity and completeness of halo samples selected by WL.

Various theoretical aspects of WL halo detection have been widely investigated by a number of authors $[8,12-16]$. The search for shear peaks in data is exemplified in some works [17-25] although, on the observational side, halo lensing studies have been mainly focused on mass determination and halo properties, e.g. density profile and concentration [26, 27].

A basic method for shear peak detection, to which we will refer in the following, is provided by the aperture mass $\left(M_{a p}\right)$ technique proposed by [28]. $M_{a p}$ is a measure of the lensing signal in a shear map smoothed with a suitable filter. The method then relies on the usual approach of searching for points of local maximum (peaks), with snr higher than a given threshold, in the smoothed map.

Here, we investigate the impact of the halo concentration on $M_{a p}$ and shear peak counts, as compared to that of some cosmological parameters (i.e. $\Omega_{m}$ and $\sigma_{8}$ ). As already noted by [29] and detailed in the following, the shear peak abundance, is quite sensitive to halo concentration and its relation with the halo mass. This seems to point out peak counts as a promising and complementary method to delineate the, still debated, relation between halo mass and concentration ( $M-c$ relation). Our main interest here is to further deepen this point quantifying the power of peak function to constrain the $M-c$ relation.

Halo concentrations have been studied extensively, by means of $\mathrm{N}$-body simulations as well as observationally, during the last decade. The general trend is that the mean halo concentration shows a strong correlation with the halo mass. Typically, it declines with increasing the mass and redshift in accordance with the idea that the central density of halos reflects the mean density of the universe at the time of their formation. Therefore, halos collapsing earlier are expected to be denser than the more massive halos collapsing later.

However, there is enough uncertainty on the exact form of the $M-c$ relation. Beside discrepancies between theoretical predictions and observations, differences are found even when comparing simulation results of different groups as well as different sets of observations [26, 30-41].

One of the main predictions of $\mathrm{N}$-body simulations is that the density profile of halos assembled hierarchically and close to virial equilibrium, can be well approximated by a Navarro, Frenk \& White (NFW) profile [42], regardless of halo mass and the details of the cosmological model, all the cosmological informations being contained in correlation between the parameters of the NFW profile, i.e. in the $M-c$ relation. The median $M-c$ relation predicted by simulations is well described by a power-law [34-36, 43, 44].

However, the variety of individual halo aggregation histories causes a scatter in concentration which can be modeled by a log-normal distribution with a variance ranging from 
$\sim 0.15$ up to $\sim 0.30$ depending on the degree of relaxation of the halo [45]. On the other hand, the redshift evolution of the concentration is less established. Recent results seem to favor a weaker redshift dependence than previously supposed [35, 36, 46].

Probably, the best current results from simulations are those by [39] and [40]. They found larger concentrations than those reported in [36] and [35], with a reasonable agreement for galaxy-size halos ( $10-15 \%$ difference) but substantially larger values $(\sim 40-$ $50 \%$ ) for cluster-size halos, more compatible with recent X-ray and kinematic observations. Furthermore, these high-resolution large-volume simulations (Bolshoi and MultiDark, see http://www.mutidark.org) seem to indicate a $M-c$ relation more complex than previously conceived showing a novel feature: at high redshift, concentration first declines with increasing mass, then flattens and increases slightly at higher masses.

On the observational side, many different methods have been used to study masses and concentrations resulting in an even more controversial picture and making the comparison between observations and theory somewhat ambiguous.

Gravitational lensing is one of the most promising methods to estimate mass and concentrations. Strong and weak lensing have been used individually or in combination. More classical methods relies on kinematic tracers, e.g. galaxies $([47,48]$ and references therein) and the hydrostatic analysis of X-ray intensity profile of halos. However, all these methods are susceptible to bias effects and are presumably affected by systematics not fully understood and/or modeled, yielding discrepant results.

For example, [37] used the stacked weak lensing signal from galaxies, groups and clusters in the Sloan Digital Sky Survey (SDSS) finding a $M-c$ relation slope consistent with the simulations but a $2 \sigma$ lower normalization. [38] performed a combined weak and strong lensing analysis of a sample of clusters, reporting concentrations with a $7 \sigma$ excess above the simulation predictions. [26] performed a weak lensing analysis of X-ray selected clusters reporting a slope somewhat steeper than in simulations although with an error of $\sim 50 \%$. Other studies based on lensing and X-ray data can be found in [32, 49-54].

Despite the qualitative agreement with simulation predictions, the general picture which emerges from observations can be summarized briefly as follows. In almost all cases, the observed slope of the $M-c$ relation is consistent with or steeper than theoretical predictions. Moreover, strong lensing measures of massive clusters and X-ray analysis give a normalization factor higher than predicted by simulations, strong lensing concentrations being systematically larger than X-ray concentrations. On the other hand, weak lensing methods seem to point out a normalization lower than that found in simulations.

For what concerns the lensing measurements, the origin of these discrepancies should be searched in some orientation and shape biases [55-57]. It has been shown that neglecting halo triaxiality can lead to over- and under-estimates of a factor of 2 in concentrations and up to $50 \%$ in halo mass other than underestimation of statistical uncertainties [58]. In addition, as already outlined above, projection of structures along the line-of-sight can result in apparently high concentration (e.g. [59]).

Further complications are due to baryonic processes. Although baryon physics is not expected to drive the structure formation process on very large scales, its effects are likely to be important for low-mass halos or in the central region of larger objects. It has been shown that baryonic feedback and cooling can alter halo profiles. In particular, baryonic cooling (see [60] and reference therein) can be responsible for the excess in concentration observed in groups and low-mass clusters [61, 62].

Dynamical and $\mathrm{X}$-ray techniques assume the halos are in virial and/or hydrostatic equi- 
librium. Most of real halos are not relaxed and have complex substructures making measurements difficult to interpret. Mergers, active galactic nuclei, cosmic rays, magnetic fields, turbulence and bulk motion of the gas can compromise the hydrostatic equilibrium leading to underestimates of X-ray masses [63-67].

Although recent hydrodynamical simulations include a number of these non-gravitational processes, they are very difficult to model. Furthermore, many of them take place at scales too small to be resolved by simulations demanding radical simplifications and approximations.

It is also worth noticing that the above results were derived in the contest of the standard cosmological model $(\Lambda \mathrm{CDM})$. Discrepancies can also arise if the data-model comparison has been made for the wrong cosmology. A number of studies have shown that alternative DE cosmologies or modified gravity models can lead to a different evolution of matter perturbations affecting the halo concentrations. For example, scalar field DE models with an earlier structure formation resulting in more concentrated halos have been considered in [43, 68-72].

The paper is organized as follows: the $M_{a p}$ method for shear peak detection is reviewed in Section 2 while in Section 3 we investigate the impact of the $M-c$ relation on the probability distribution function of $M_{a p}$ and provide a prescription to analytically calculate it taking into account the dispersion in $M-c$ relation. The impact on peak number counts is then investigated and compared to that of $\Omega_{m}$ and $\sigma_{8}$. In Section 4 we perform a Fisher matrix analysis in order to assess the capability of upcoming weak lensing survey in constraining the $M-c$ relation by means of peak counts. Section 5 is devoted to discussion and conclusions.

\section{Aperture Mass}

The gravitational field of a sufficiently high matter concentration causes a coherent shape distortion of the images of faint background galaxies. Measurements of these shear distortions, would thus permit to detect massive halos by searching for peaks in wide-field shear maps. To this aim, different WL methods for halo detection, based on linear filtering techniques, have been developed in the last decade [12, 28, 73-75] (for alternative approaches see [76-78]).

Here, we use the aperture mass, $M_{a p},[28]$ as peak finder. $M_{a p}$, is the projected mass distribution, or convergence $\kappa$, smoothed on the angular scale $\vartheta_{0}$ :

$$
M_{a p}\left(\vartheta_{0}\right)=\int d^{2} \theta \kappa(\boldsymbol{\theta}) U(|\boldsymbol{\theta}|)
$$

where $\boldsymbol{\theta}=(\vartheta \cos \phi, \vartheta \sin \phi)$ and $U(\vartheta)$ is a compensated filter function, i.e. $\int_{0}^{\vartheta_{0}} d \vartheta \vartheta U(\vartheta)=0$, which vanishes for $\vartheta>\vartheta_{0}$. The main advantages in using compensated filters are that $M_{a p}$ is not influenced by mass-sheet degeneracy and the possibility of expressing $M_{a p}$ in terms of the (observable) tangential shear $\gamma_{t}$ :

$$
M_{a p}\left(\vartheta_{0}\right)=\int d^{2} \theta \gamma_{t}(\boldsymbol{\theta}) Q(|\boldsymbol{\theta}|)
$$

where $\gamma_{t}(\boldsymbol{\theta})=-\operatorname{Re}\left[\gamma(\boldsymbol{\theta}) e^{-2 i \phi}\right]$ is the tangential component of the shear at position $\boldsymbol{\theta}$ and $Q$ is a filter function related to $U$ by:

$$
Q(\vartheta)=\frac{2}{\vartheta^{2}} \int_{0}^{\vartheta} d \vartheta^{\prime} \vartheta^{\prime} U\left(\vartheta^{\prime}\right)-U(\vartheta)
$$




\subsection{Filter function}

Several filter functions $Q(\vartheta)$ have been considered depending on their specific application in WL studies (cosmic shear, halo searching, etc.). The ideal choice for radially symmetric halos, would be a filter profile proportional to the shear profile, i.e. $Q(\vartheta) \propto \gamma(\vartheta)$ thus to maximize the signal-to-noise ratio snr associated to halo detections as shown in [28]. Some examples of filters can be found in [79] (polynomial filter), [12] (Gaussian filter), [74] (optimized for NFW halo and LSS noise suppression), [80] (non-parametric filter).

To our aim, a reasonable choice is the filter introduced by [73] and optimized for NFW halos, approximating their shear signal with a hyperbolic tangent:

$$
Q(x)=\left[\pi \vartheta_{0}\left(1+e^{a-b x}+e^{-c+d x}\right)\right]^{-1} \frac{\tanh \left(x / x_{c}\right)}{\left(x / x_{c}\right)}
$$

where $x=\vartheta / \vartheta_{0}$. The values $a=6, b=150, c=47$ and $d=50$ are chosen in order to have exponential cut-offs at small and large radii while $x_{c}=0.1$ is a good choice for the filter profile slope as empirically shown by [17]. The related filter $U(\vartheta)$ is obtained following the procedure described in [14]. In the following we will always set $\vartheta_{0}=7^{\prime}$. We have checked that this choice is the best compromise which maximizes the number of detections over the redshift range of our interest.

\subsection{Halo profile and mass-concentration relation}

In order to compute the convergence $\kappa$ of cluster-sized halos entering in (2.1), we model their density profile by a Navarro, Frenk \& White (NFW) profile:

$$
\rho(r)=\frac{\rho_{s}}{\left(r / r_{s}\right)\left(1+r / r_{s}\right)^{2}}
$$

The finite extent of halos is taken into account by truncating the profile at the radius $r_{200}$, within which the average density, $\Delta_{200} \rho_{c r}$, is 200 times the critical density of the universe $\rho_{c r}=3 H^{2} / 8 \pi G$ ( $H$ and $G$ being the Hubble parameter and the Newton's gravitational constant respectively).

The scale radius $r_{s}$ is related to $r_{200}$ through the concentration parameter $c_{200}=r_{200} / r_{s}$ giving:

$$
\frac{\rho_{s}}{\rho_{c r}}=\frac{1}{3} \Delta_{200} f_{200}
$$

where:

$$
f_{200}=c_{200}^{3}\left[\ln \left(1+c_{200}\right)-\frac{c_{200}}{1+c_{200}}\right]^{-1}
$$

The halo mass is then defined as:

$$
M_{200}=\frac{4 \pi}{3} \Delta_{200} \rho_{c r} r_{200}^{3}=4 \pi \rho_{s} r_{200}^{3} f_{200}^{-1}
$$

A number of heuristic models for the median mass-concentration relation, calibrated against simulations, have been suggested [30, 31, 42, 44]. Here we assume the common simple relation:

$$
c_{200}\left(M_{200}, z\right)=c_{0}\left[\frac{M_{200}}{M_{0}}\right]^{\alpha} g(z)
$$

with the concentration growth factor, $g(z)$, as proposed by [36] and we set $M_{0}=10^{14} h^{-1} M_{\odot}$. Their model is a modification of the commonly used Bullock model [30], based on the simple 
assumption that the characteristic density of the halo, $\rho_{s}$, remain constant after the halo forms. This results in a concentration growth factor:

$$
g(z)=\left[\frac{H_{0}}{H(z)}\right]^{2 / 3}=\left[\frac{\rho_{c r, 0}}{\rho_{c r}(z)}\right]^{1 / 3}
$$

rather than $g(z)=1 /(1+z)$ of the original Bullock model. The normalization of the $M-c$ relation $c_{0}$ is thus defined as the concentration $c_{200}$ of a halo with mass $M_{200}=10^{14} h^{-1} M_{\odot}$ at $z=0$. Note that (2.10) seems more consistent with the idea that halo concentrations are related to the ratio of the present background density, $\rho_{c r, 0}$, and that at the halo formation epoch. It reduces to the Bullock model in the case of Einstein-De Sitter universe and is able to reproduce the $M-c$ relation, in a $\Lambda C D M$ universe, over a wide range of masses $\left(10^{10} h^{-1} M_{\odot} \lesssim M \lesssim 10^{15} h^{-1} M_{\odot}\right)$ probed by simulations. Its scaling with $\rho_{c r}$, however, would suggest that it could be more suitable than the Bullock model in describing the massconcentration evolution in cosmologies different from $\Lambda C D M$. For simplicity, we assume a constant slope $\alpha$ over the mass range considered although simulations indicate a slight deviation at higher masses.

The convergence for a truncated NFW profile then reads [12]:

$$
\kappa\left(\vartheta, z_{h}, z_{g}\right)=\frac{8 \pi G}{c^{2}} \rho_{s} r_{s} \frac{D_{h} D_{h g}}{D_{g}} f(x),
$$

where $x=\vartheta / \vartheta_{s}, \vartheta_{s}=r_{s} / D_{h}, D$ denotes the angular diameter distance, the subscripts $h$ and $g$ stand for lens and source galaxies respectively. Finally, the function $f$ is given by:

$$
f(x)=\left\{\begin{array}{lr}
-\frac{\sqrt{c_{200}-x^{2}}}{\left(1-x^{2}\right)\left(1+c_{200}\right)}+\left(1-x^{2}\right)^{-3 / 2} \operatorname{arccosh} \frac{x^{2}+c_{200}}{x\left(1+c_{200}\right)} & x<1 \\
\sqrt{c_{200}^{2}-1} \frac{\left(2+c_{200}\right)}{3\left(1+c_{200}\right)^{2}} & x=1 \\
-\frac{\sqrt{c_{200}-x^{2}}}{\left(1-x^{2}\right)\left(1+c_{200}\right)}-\left(x^{2}-1\right)^{-3 / 2} \arccos \frac{x^{2}+c_{200}}{x\left(1+c_{200}\right)} & 1<x \leq c_{200} \\
0 & x>c_{200}
\end{array}\right.
$$

\subsection{Galaxy survey}

If the source galaxies are distribute in redshift, the convergence $\kappa$ entering in (2.1) is the convergence averaged over the normalized source-redshift distribution $p\left(z_{g}\right)\left(z_{g}\right.$ being the redshift of the source galaxies). For a NFW halo at a redshift $z_{h}$ with convergence $\kappa\left(\vartheta, z_{h}, z_{g}\right)$ then we have:

$$
\kappa(\vartheta)=\int_{z_{h}}^{\infty} d z_{g} p\left(z_{g}\right) \kappa\left(\vartheta, z_{h}, z_{g}\right)
$$

Here, we assume a distribution $p(z)$ of the standard form:

$$
p(Z)=\frac{\beta}{z_{0} \Gamma\left(\frac{a+1}{b}\right)} Z^{a} e^{-Z^{b}}
$$

where $Z=z / z_{0}$ and we set $a=2, b=1.5$ and $z_{0}=0.6$. We further assume an ellipticity dispersion of the sources $\sigma_{\epsilon}=0.3$, a galaxy number density $n=30 \mathrm{gal} / \mathrm{arcmin}^{2}$ and a survey area of $15000 \mathrm{deg}^{2}$. With these values (2.13) provides a galaxy distribution as expected from the Euclid survey, with a mean redshift $z_{\text {mean }}=0.9[4,81]$. 


\begin{tabular}{|cccccc||ccc|}
\hline \multicolumn{7}{|c|}{$\begin{array}{c}\text { Cosmological parameters } \\
\text { WMAP9 }+ \text { SNLS3 }+ \text { BAO }\end{array}$} & \multicolumn{3}{|c}{$\begin{array}{c}M-c \text { relation } \\
\text { parameters }\end{array}$} \\
\hline$\Omega_{m}$ & $\Omega_{b}$ & $\sigma_{8}$ & $H_{0}$ & $\tau$ & $n_{s}$ & $c_{0}$ & $\alpha$ & $\sigma_{\ln c}$ \\
\hline 0.291 & 0.047 & 0.828 & 68.98 & 0.087 & 0.969 \\
\hline
\end{tabular}

Table 1. Fiducial cosmological and $M-c$ relation parameters.

\section{Peak function}

In real observations, the measured value of $M_{a p}$ will differ from the real one due to several effects. Statistical noise and halo shape induce scatter and bias in $M_{a p}$ and hence in the resulting shear peak counts. A detailed analysis on how noise and the halo structure affect the probability distribution function (PDF) of $M_{a p}$ and the peak counts was performed in $[12,82]$ (and references therein, see also [83] and [84]) employing both analytical description of halos and mock data of WL survey generated from numerical simulations. Statistical noise due to intrinsic ellipticities, survey shot noise and projection effects of large scale structure (LSS) along the line-of-sight, affect the peak heights and generate spurious peaks in the shear maps resulting in an excess in real counts over the theoretical predictions.

On the other hand, the dark matter distribution in real halo is not spherically symmetric but highly elongated [85] so that deviations from the universal NFW density profile are expected. Furthermore, simulation results tell us that halo concentrations are log-normally distributed among halos with a given mass, with variance $\sim 0.15-0.3$ [45].

All these effects have a large impact on WL measurements of halos and induce scatter and bias in the peaks heights therefore affecting the peak counts. For simplicity, we do not consider here deviation from sphericity and assume halos to have NFW profiles while we only focus on the effects due to statistical noise and, in particular, concentration scatter. Impact of halo triaxiality on WL peak searches was accurately studied in [82].

In order to predict the expected abundance of halo peaks we need to know the PDF of $M_{a p}$ and the halo mass function. As a fiducial model we assume a $\Lambda$ CDM cosmology with cosmological parameters set to the best fit values estimated from nine-years WMAP data in conjunction with the Supernova Legacy Survey three-year sample (SNLS3) and baryon acoustic oscillations (BAO) measurements [86]. Fiducial cosmological parameters are summarized in table $1\left(\Omega_{b}, H_{0}, \tau\right.$ and $n_{s}$ denoting the baryon density parameter, the present value of the Hubble parameter, the optical depth to reionization and the spectral index of primordial scalar fluctuations) together with the assumed fiducial parameters for the $M-c$ relation.

\section{1 $M_{a p}$ probability distribution function}

A significant part of the noise contribution to $M_{a p}$ comes from intrinsic ellipticity and the finite number of background galaxies used to measure the shear signal. Nevertheless, the accuracy with which the lensing signal of individual halos can be measured is also limited by the presence of large-scale structures (LSS) along the line-of-sight, representing an additional statistical source of noise as discussed in [9, 74] (see also [79]).

Here we neglect the effects on $M_{a p}$ measurements due to intrinsic alignments of background galaxies and LSS projections correlated with halo lens (although they might introduce non-negligible bias and noise as discussed in $[80,87]$ ) and we mainly focus on the impact of 
the concentration scatter and of the statistical noise from uncorrelated intrinsic ellipticities and uncorrelated LSS.

Following [74] we roughly split the total signal in two independent contributions: the signal from nonlinear scales due exclusively to massive halos and the LSS signal generated by linearly evolved matter perturbations. Although questionable for many WL applications, it would be sufficient for our purposes. The observed $M_{a p}$ is then given by the sum of halo and LSS signals plus the noise contribution $M_{a p}^{g}$ due to intrinsic ellipticity of the galaxies and survey shot noise:

$$
M_{a p}=M_{a p}^{\text {halo }}+M_{a p}^{L S S}+M_{a p}^{g}
$$

Under the assumption of uncorrelated intrinsic ellipticities and uncorrelated LSS both galaxy and LSS noise fields can be modeled as isotropic Gaussian random fields with zero mean, $\left\langle M_{a p}^{g}\right\rangle=\left\langle M_{a p}^{L S S}\right\rangle=0$, and variances given respectively by (see [9, 74, 79] for the derivation):

$$
\sigma_{g}^{2}\left(\vartheta_{0}\right)=\frac{\pi \sigma_{\epsilon}^{2}}{n} \int_{0}^{\vartheta_{0}} d \vartheta \vartheta Q^{2}(\vartheta)
$$

( $\sigma_{\epsilon}$ is the ellipticity dispersion of sources, $n$ is the average number density of galaxies inside the aperture), and:

$$
\sigma_{L S S}^{2}\left(\vartheta_{0}\right)=2 \pi \int_{0}^{\infty} d l l P_{\kappa}(l) g^{2}\left(l, \vartheta_{0}\right)
$$

Here, the function $g\left(l, \vartheta_{0}\right)$ reads:

$$
g\left(l, \vartheta_{0}\right)=\int_{0}^{\vartheta_{0}} d \vartheta \vartheta U(\vartheta) J_{0}(l \vartheta)
$$

while the power spectrum of the LSS convergence $P_{\kappa}(l)$ is related to the power spectrum of the three-dimensional density fluctuations $P_{\delta}(k)$ by Limber's equation:

$$
P_{\kappa}(l)=\frac{9 H_{0}^{2} \Omega_{m}^{2}}{4 c^{2}} \int_{0}^{\chi_{h}} d \chi \frac{W^{2}(\chi)}{a^{2}(\chi)} P_{\delta}\left(\frac{l}{D(\chi)}, \chi\right)
$$

( $\chi$ and $D(\chi)$ being the comoving distance and the comoving angular diameter distance respectively). For a given distribution of source galaxies, $p(\chi) d \chi=p(z) d z$, the weight function $W$ reads:

$$
W(\chi)=\int_{\chi}^{\chi_{h}} d \chi^{\prime} p(\chi) \frac{D\left(\chi^{\prime}-\chi\right)}{D\left(\chi^{\prime}\right)}
$$

where $\chi_{h}$ is the distance to the last scattering surface.

The Gaussian noise $\sigma_{n}^{2}=\sigma_{g}^{2}+\sigma_{L S S}^{2}$ then leads to a probability distribution function (PDF) for $M_{a p}$ given by:

$$
p_{n}\left(M_{a p} \mid M\right)=\frac{1}{\sqrt{2 \pi} \sigma_{n}} \exp \left[-\frac{1}{2}\left(\frac{M_{a p}-M_{a p}^{\text {halo }}(M)}{\sigma_{n}}\right)^{2}\right]
$$

where $M_{a p}^{\text {halo }}(M)$ is the true value of the aperture mass of a halo with mass $M$.

Although observations and simulation results tell us that, for a given mass $M$, halo concentrations are $\log -$ normally distributed with dispersion $\sigma_{\ln c} \sim 0.15-0.3$ [45]:

$$
p(c \mid M)=\frac{1}{c \sqrt{2 \pi} \sigma_{\ln c}} \exp \left[-\frac{1}{2}\left(\frac{\ln c-\ln \hat{c}(M)}{\sigma_{\ln c}}\right)^{2}\right]
$$


$(\hat{c}(M)$ being the median concentration), it is common practice in theoretical calculations to use the PDF (3.7) where $M_{a p}^{\text {halo }}$ is calculated assuming the median $M-c$ relation and neglecting any dispersion (hereafter, we will drop the subscript 200 to denote the halo concentration).

In order to take the concentration scatter into account, one should instead consider the convolution:

$$
p\left(M_{a p} \mid M\right)=\int d M_{a p}^{\text {halo }} p_{n}\left(M_{a p} \mid M, c\right) p_{c}\left(M_{a p}^{h a l o} \mid M\right)
$$

where:

$$
p_{c}\left(M_{a p}^{\text {halo }} \mid M\right) d M_{a p}^{\text {halo }}=p(c \mid M) d c
$$

and now $M_{a p}^{\text {halo }}(M)=M_{a p}^{\text {halo }}(M, c)$. It is then easy to show with a little algebra that the mean value of $M_{a p}$ is:

$$
\left\langle M_{a p}\right\rangle=\int d M_{a p} M_{a p} p\left(M_{a p} \mid M\right)=\int d M_{a p}^{\text {halo }} M_{a p}^{\text {halo }} p_{c}\left(M_{a p}^{\text {halo }} \mid M\right)=\left\langle M_{a p}^{\text {halo }}\right\rangle
$$

while the total $M_{a p}$ variance $\sigma$ reads:

$$
\begin{aligned}
\sigma^{2} & =\int d M_{a p}\left(M_{a p}-\left\langle M_{a p}\right\rangle\right)^{2} p\left(M_{a p} \mid M\right) \\
& =\int d M_{a p}^{\text {halo }}\left(M_{a p}^{\text {halo }}-\left\langle M_{a p}^{\text {halo }}\right\rangle\right)^{2} p_{c}\left(M_{a p}^{\text {halo }} \mid M\right)+\sigma_{n}^{2} \\
& =\sigma_{c}^{2}+\sigma_{n}^{2}
\end{aligned}
$$

Note that, because of the non-linearity of the $M-c$ relation $\left\langle M_{a p}\right\rangle=\left\langle M_{a p}^{\text {halo }}\right\rangle \neq M_{a p}^{\text {halo }}(\langle c\rangle)$ and the same holds for the median value $\hat{M}_{a p}$.

Finally, we define the signal-to-noise ratio, snr, associated to each peak detection as:

$$
s n r=\frac{\hat{M}_{a p}}{\sigma}
$$

(here we have defined $s n r$ in terms of the median $\hat{M}_{a p}$ since $p\left(M_{a p} \mid M\right)$ is expected to be non-symmetric because of the asymmetry of (3.8)).

It is worth noticing that, unlike the statistical noise $\sigma_{n}$ which is not related to halo properties, $\sigma_{c}$ depends on both lens mass and redshift $z_{h}$, it increases (decreases) with $M$ $\left(z_{h}\right)$. This is shown in fig. 1 where the different contributions to the total variance $\sigma$ are displayed as a function of $z_{h}$ for different halo masses in the range $10^{13}-5 \cdot 10^{15} h^{-1} M_{\odot}$. Inspection of the figure indicates that the scatter in $M-c$ relation affects significantly the $M_{a p}$ variance (and hence $s n r$ ) of halos with higher mass at relatively low redshifts. Its impact, however, becomes negligible at higher $z_{h}$. Fig. 2 shows the PDF $p\left(M_{a p} \mid M\right)$ obtained from (3.9) for lens masses of $10^{14}$ (left panel) and $10^{15} h^{-1} M_{\odot}$ (right panel) at $z_{h}=0.2$. For comparison we also show the PDFs in the cases when only the statistical noise is considered $\left(p_{n}\left(M_{a p} \mid M\right)\right.$, eq. (3.7)) or only the effect of $M-c$ relation scatter is taken into account $\left(p_{c}\left(M_{a p} \mid M\right)\right.$, eq. (3.10)). In the former case, $\sigma_{c} \simeq \sigma_{L S S} \ll \sigma_{g}$ so that $p \simeq p_{n}$ while in the latter $\sigma_{c}$ and $\sigma_{g}$ are of the same order of magnitude (see also fig. 1).

The distorsions in the PDF caused by variations in the parameters $c_{0}, \sigma_{\ln c}$ and $\alpha$ of the $M-c$ relation are shown in the left, central and right panels of fig. 3. The solid line indicates the fiducial model prediction. Increasing the normalization $c_{0}$ shifts the mean value $\left\langle M_{a p}\right\rangle$ to larger values while changes in $\sigma_{\ln c}$ mainly affect the width and the skewness of the distribution which is only slightly altered by variations in $\alpha$. 

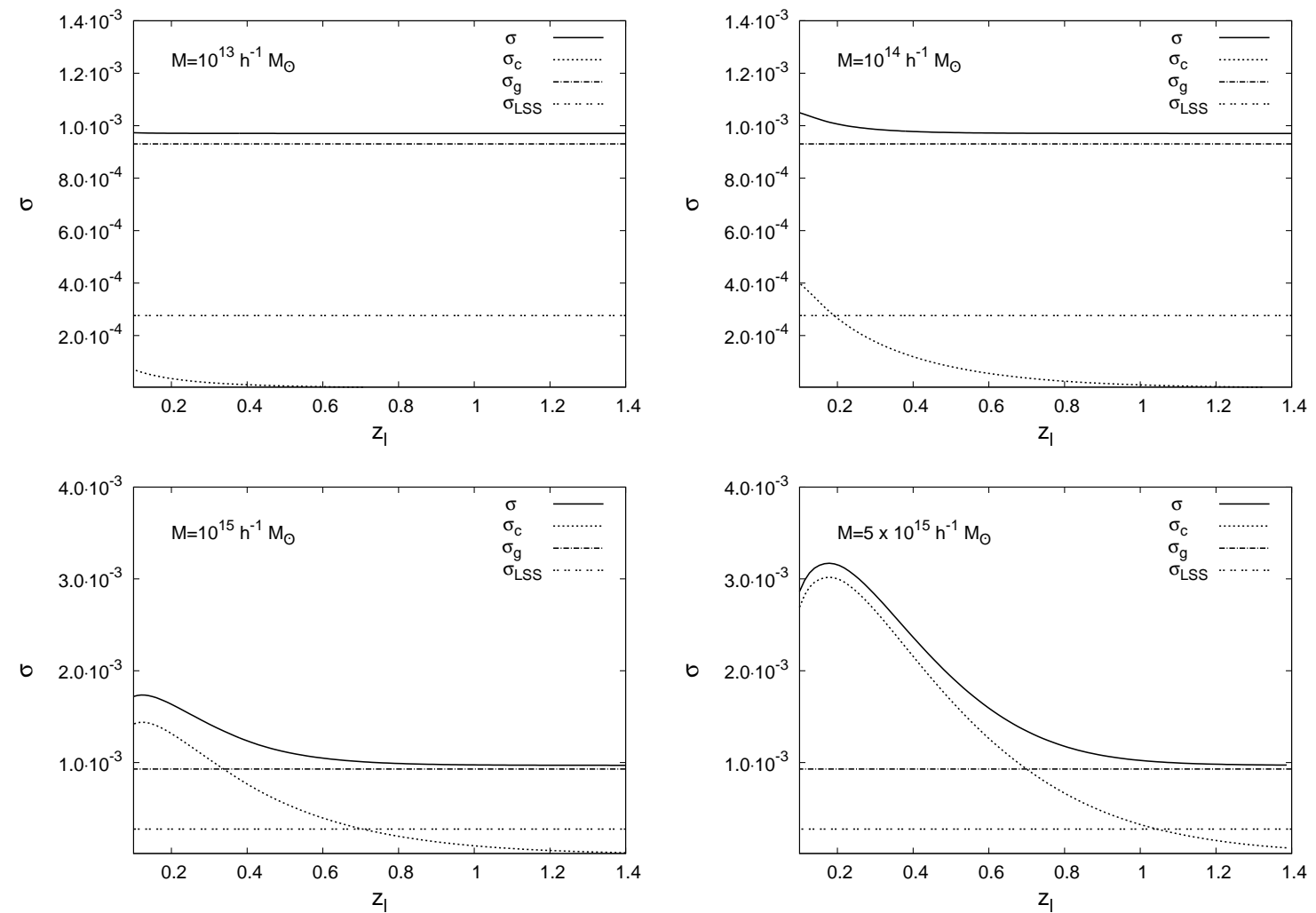

Figure 1. Contributions to the total $M_{a p}$ variance $\sigma$ as function of lens redshift and mass, due to intrinsic ellipticities and finite numer of background galaxies $\left(\sigma_{g}\right)$, LSS $\left(\sigma_{L S S}\right)$ and concentration scatter $\left(\sigma_{c}\right)$.

The dependence of snr on lens mass and redshift is shown in fig. 4. In the left panel only statistical noise is considered while in the central panel we also include the concentration scatter. The right panel displays the relative deviation $\Delta s n r / s n r$ between them. As discussed above, concentration scatter significantly increases the $M_{a p}$ variance of more massive halos. In turn, the associated snr is strongly suppressed to no more than $s n r=10$.

We have also checked that shifts in the cosmological parameters $\sigma_{8}, \Omega_{m}, H_{0}$ and $n_{s}$ do not appreciably modify the $M_{a p}$ PDF and $s n r$. This fact that they are almost independent of the underlying cosmology, can be understood looking at how cosmology enters in the evaluation of $M_{a p}$ and $\sigma$. The convergence $\kappa(\vartheta)$ depends on the integrated Hubble rate through the lensing efficiency function $D_{h} D_{h g} / D_{g}$ which only weakly depends on cosmology since it involves a ratio of distances which is then further integrated over the source redshift. Further, $M_{a p}$ is independent of $n_{s}$ and $\sigma_{8}$. On the other hand, $n_{s}$ and $\sigma_{8}$ affect the LSS convergence power spectrum $P_{\kappa}(l)$ entering the total noise $\sigma$ through the $\sigma_{L S S}$ term. Nevertheless, this latter is always about one order of magnitude or more smaller than $\sigma_{g}$, yielding a negligible effect on snr.

\subsection{Halo mass function}

Different semianalytic approaches and fits to simulation results, aimed to model the abundance of halos [88-92], can be characterized by the scaled differential halo mass function 

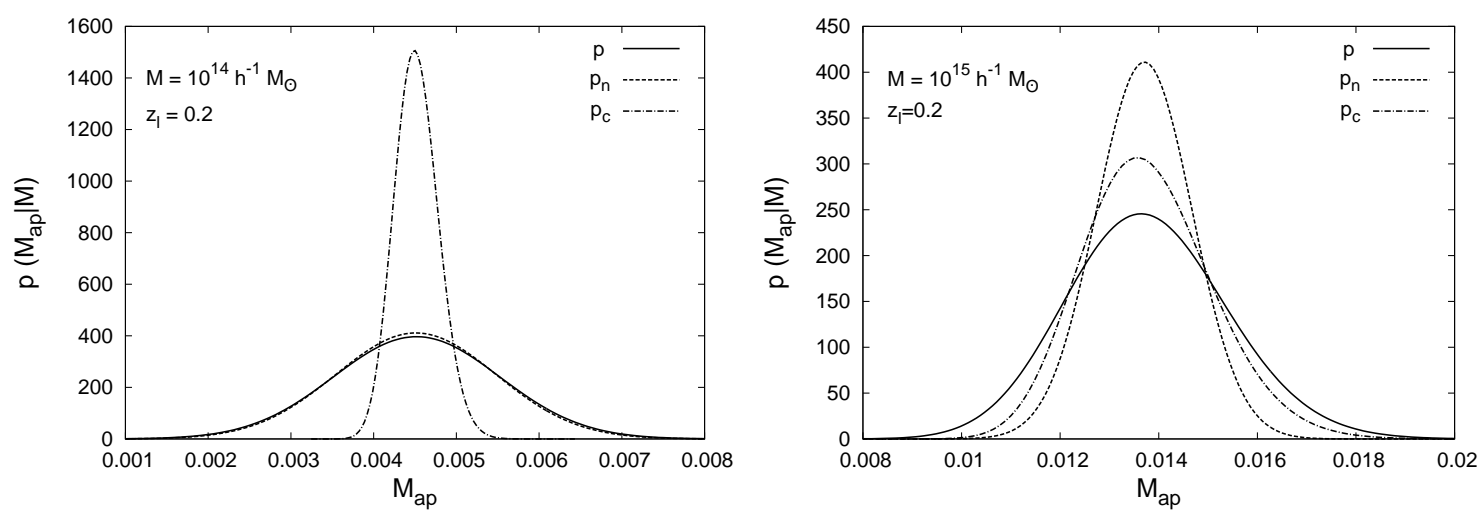

Figure 2. PDF $\left(p\left(M_{a p} \mid M\right)\right)$ for lens masses of $10^{14}$ (left panel) and $10^{15} h^{-1} M_{\odot}$ (right panel) at $z_{h}=0.2$ compared to the PDFs in the cases when only the statistical noise is considered $\left(p_{n}\left(M_{a p} \mid M\right)\right)$, or only the effect of $M-c$ relation scatter is taken into account $\left(p_{c}\left(M_{a p} \mid M\right)\right)$.
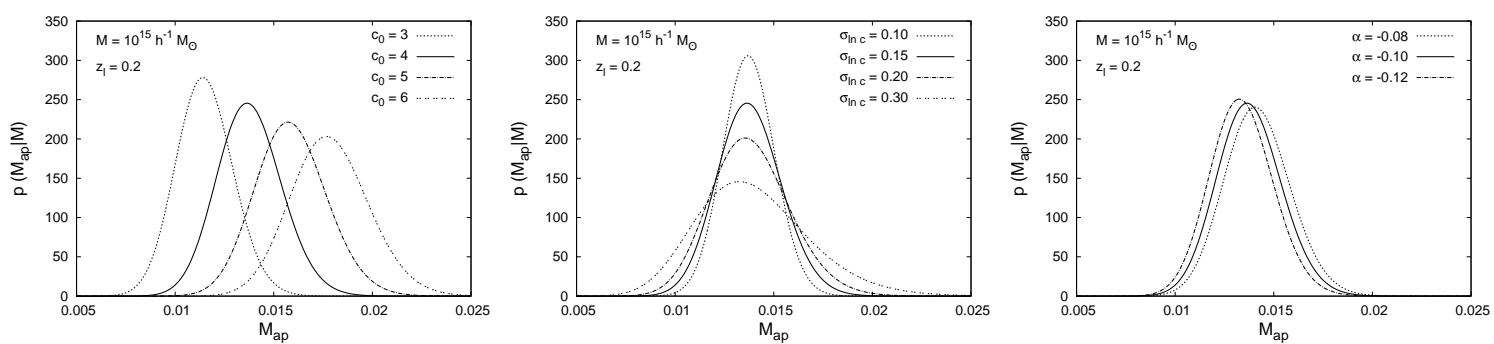

Figure 3. Effect of varying the $M-c$ relation parameters, $c_{0}$ (left panel), $\sigma_{\ln c}$ (central panel) and $\alpha$ (right panel), on $M_{a p}$ PDF. Results are shown for an halo of mass $M=10^{14} h^{-1} M_{\odot}$ located at $z_{h}=0.2$.
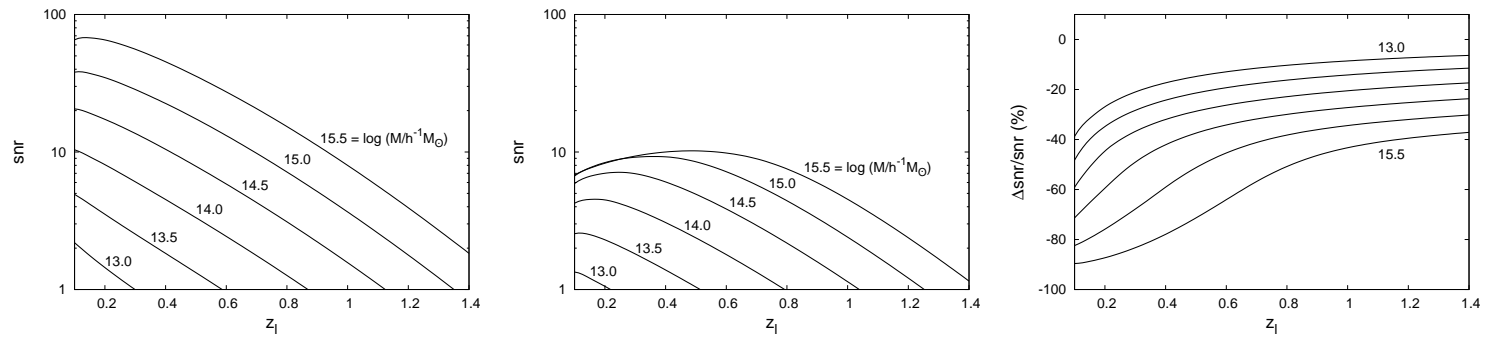

Figure 4. Dependence of snr on lens mass and redshift $z_{h}$. In the left panel only statistical noise is considered while in central panel the concentration scatter is also taken into account. The relative deviation of $s n r$ between the two cases is shown in the right panel. Results are given for masses in the range $10^{13}-5 \cdot 10^{15} h^{-1} M_{\odot}$ in steps of $\Delta \log M=0.5$.

introduced in [90]:

$$
f\left(\sigma_{M}, z\right) \equiv \frac{M}{\rho_{m}(z)} \frac{d n_{\text {halo }}(M, z)}{d \ln \sigma_{M}^{-1}}
$$


where $n_{\text {halo }}(M, z)$ is the number density of halos with mass $M, \rho_{m}$ is the background matter density and $\sigma_{M}=\sigma_{M}(M, z)$ is the variance of the linear density field on the scale $M$. This definition of mass function has the advantage that to a good accuracy it does not explicity depend on redshift, matter power spectrum or cosmological parameters, their dependence being all enclosed in $\sigma_{M}$. Hence, this "universality" of the mass function suggests that the form of $f$ should hold in all Gaussian hierarchical clustering model, at all times and in any cosmology. Indeed, this has been verified to a good accuracy in several works by means of $\mathrm{N}$-body simulations for a vast class of cosmologies (see, however, [93] for a recent discussion about the topic of the universality of the mass function).

For its simplicity, in the following, we make use of the fitting form provided in [90]:

$$
f\left(\sigma_{M}\right)=0.315 \exp \left[-\left|\ln \sigma_{M}^{-1}+0.61\right|^{3.8}\right]
$$

\subsection{Peak number counts}

Having determined the theoretical mass function and having detailed how the $M_{a p} \mathrm{PDF}$ and the $s n r$ for peak detection can be computed, we can now estimate the abundance of halos that produce significant peaks in the aperture mass map.

Noticing that the probability for a halo of mass $M$ to be detected as a shear peak with $s n r$ above the threshold $s n r^{*}=\hat{M}_{a p}^{*} / \sigma$ is:

$$
P\left(>s n r^{*} \mid M\right)=\int_{M_{a p}^{*}} d M_{a p} p\left(M_{a p} \mid M\right)
$$

the shear peak function expected to be observed then reads:

$$
N_{\text {halo }}\left(>s n r^{*}\right)=\int d V \int d M \frac{d n_{\text {halo }}\left(M, z_{h}\right)}{d M} P\left(>s n r^{*} \mid M\right)
$$

where $d V$ is the comoving volume element per unit solid angle ${ }^{8}$. The above expression gives the observed number of halo peaks taking into account, through (3.9), the scatter in $M_{a p}$ due to statistical noise and concentration distribution.

The total number of observed shear peaks is, however, larger than $N_{\text {halo }}$ because of spurious peaks caused by projection effects of LSS as well as shape and shot noise of background galaxies.

An analytic approach suitable to quantify the level of spurious detections, has been developed and applied in [14]. Analytic results were there compared to peak counts from numerical ray-tracing simulations finding good agreement for different filter functions. We remind here that this approach relies on peak counts in random Gaussian fields [94], very well representing noise and LSS but not non-linear objects such as virialized halos which are highly non-Gaussian. We also remind that, following [74] and according to eq. (3.1), we define the LSS signal as being due to linearly evolved structures alone so that a Gaussian description applies (see section 3.1).

According to [14], the number of spurious peaks may be estimated as:

$$
N_{s p}\left(>s n r^{*}\right)=\frac{1}{(2 \pi)^{\frac{3}{2}}}\left(\frac{\sigma_{1}}{\sigma_{n}}\right)^{2} s n r^{*} \exp \left[-\frac{s n r^{* 2}}{2}\right]
$$

\footnotetext{
${ }^{8} d V=d \chi \chi^{2}=d z\left[\int_{0}^{z} d z^{\prime} / H\left(z^{\prime}\right)\right]^{2} d \chi / d z$ in spatially flat cosmologies, $\chi$ being the comoving distance.
} 


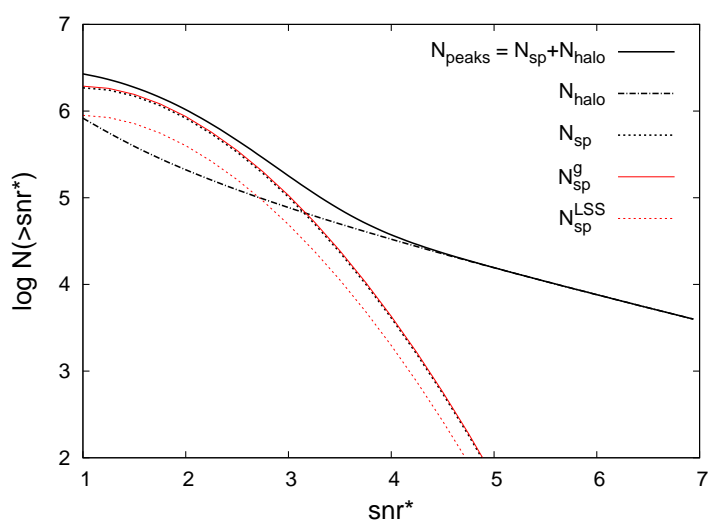

Figure 5. Number of detectable peaks as a function of $s n r^{*}$ for the fiducial model. Black lines refer to total number (solid) and the contribution from halo ( dash-dotted) and spurious (dotted) peaks. Red lines refer to spurious peaks generated by galaxy noise (solid) and LSS (dotted).
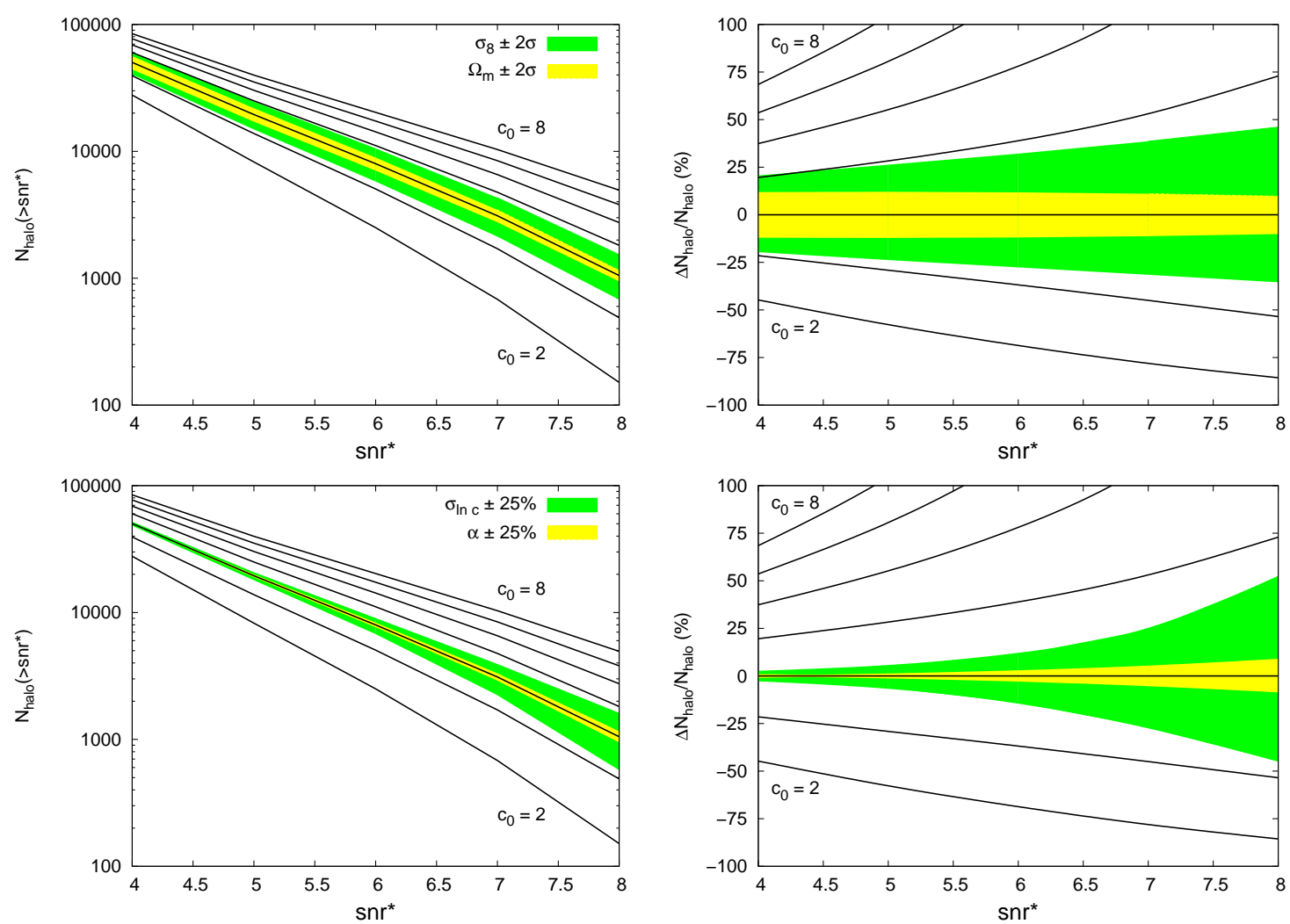

Figure 6. Left panels: Total number of halo peaks as a function of snr* in the redshift range $\Delta z=0.1-1.4$. Shaded regions indicate deviations from the fiducial model predictions arising from shifts in $\sigma_{8}$ and $\Omega_{m}$ within the current $2-\sigma$ bounds (top panels), and $\pm 25 \%$ offsets in $\alpha$ and $\sigma_{\ln c}$ (bottom panels). Solid lines refer to changes in $c_{0}$ (from 2 to 8 in steps of 1 from the lowest to highest curve). Right panels: Relative deviation $\Delta N_{\text {halo }} / N_{\text {halo }}=\left[N_{\text {halo }}-N_{\text {halo, fid }}\right] / N_{\text {halo,fid }}$. 

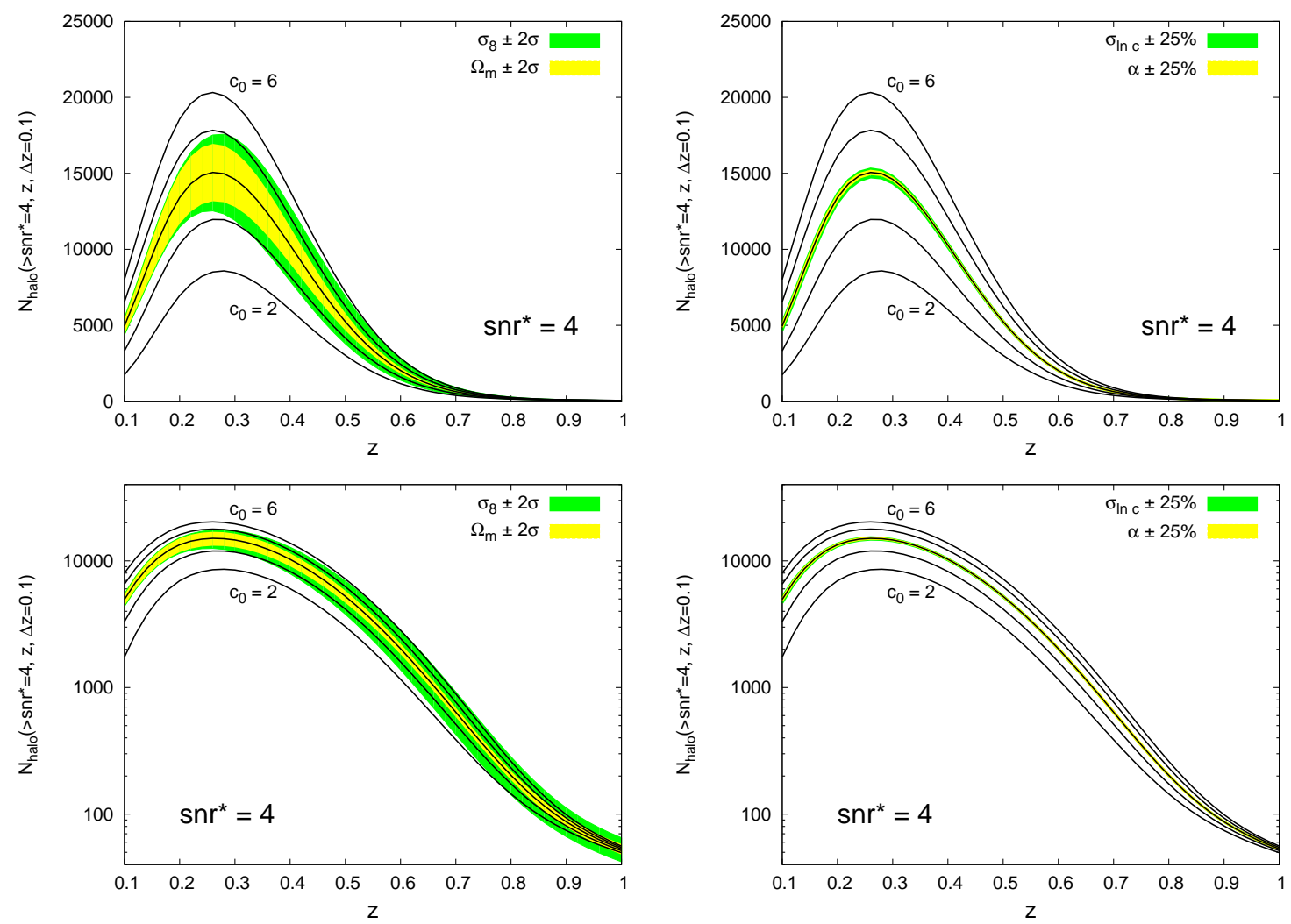

Figure 7. Top panels: Number of halo peaks in redshift bin of $\Delta z=0.1$ as a function of $z$ for $s n r^{*}=4$. Shaded regions indicate deviations from the fiducial model predictions arising from shifts in $\sigma_{8}$ and $\Omega_{m}$ within the current $2-\sigma$ bounds (left panel), and $\pm 25 \%$ offsets in $\alpha$ and $\sigma_{\ln c}$ (right panel). Solid lines refer to changes in $c_{0}$ (from 2 to 6 in steps of 1 from the lowest to highest curve). Bottom panels: Same as top panels but plotted in logarithmic scale to emphasize the behavior at high redshifts.

where:

$$
\sigma_{1}^{2}=\int d l l^{3} P(l)|\hat{Q}(\vartheta)|^{2}
$$

Here $\hat{Q}(\vartheta)$ is the Fourier transform of the filter while $P(l)=P_{n}+P_{\gamma}(l)$ is the power spectrum of the statistical noise $\left(P_{n}=\sigma_{\epsilon}^{2} / 2 n_{g a l}\right.$ and $P_{\gamma}(l)=P_{\kappa}(l) / 2$ being the power spectra of galaxy noise and LSS shear respectively).

The total number of observed peaks is finally given by:

$$
N_{\text {peaks }}\left(>s n r^{*}\right)=N_{\text {halo }}\left(>s n r^{*}\right)+N_{s p}\left(>s n r^{*}\right)
$$

Fig. 5 shows $N_{\text {peaks }}, N_{\text {halo }}$ and $N_{s p}$ as a function of the threshold $s n r^{*}$ for our fiducial model. Spurious peaks generated by galaxy noise alone $N_{s p}^{g}$ and LSS alone $N_{s p}^{L S S}$ are also shown for comparison.

It is worth noticing that the abundance of spurious detections (although it can vary with the filter and/or the filter scale, see [14]) is almost independent of the underlying cosmology, the only dependence on cosmology being ascribed to the LSS power spectrum $P_{\kappa}(l)$ entering the ratio $\sigma_{1} / \sigma_{n}$. Indeed, as observed at the end of section 3.1 (see also fig. 1), the LSS 

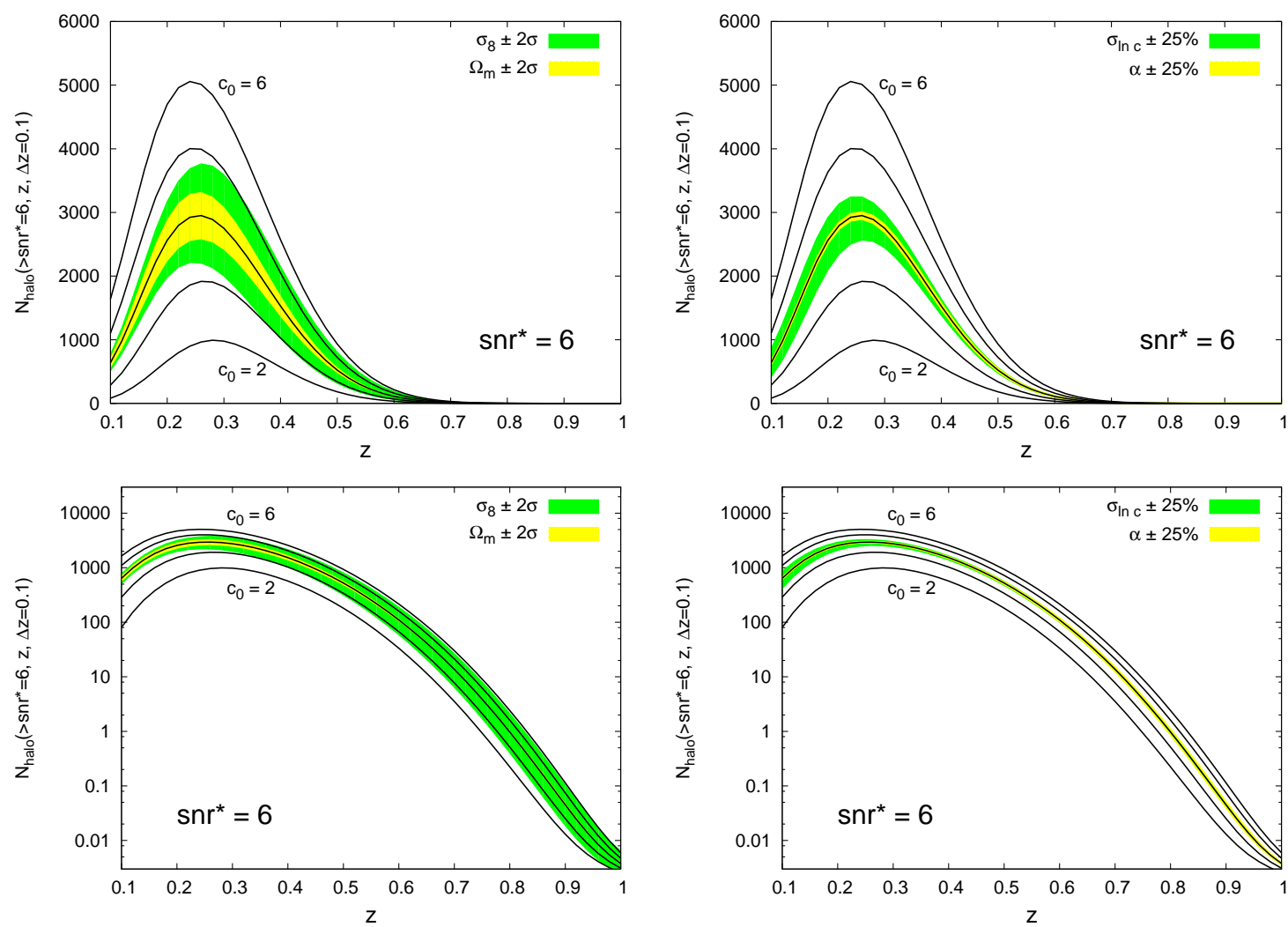

Figure 8. As in previous figure but for $s n r^{*}=6$.

contribution to $\sigma_{n}^{2}=\sigma_{g}^{2}+\sigma_{L S S}^{2}$ is always about one order of magnitude smaller than the galaxy noise contribution and the same holds for $\sigma_{1}^{2}=\sigma_{1, g}^{2}+\sigma_{1, L S S}^{2}$. Hence, $N_{s p} \simeq N_{s p}^{g}$ (see fig. 5) and the cosmological dependence is strongly suppressed. We have checked that no substantial changes in the number of spurious peaks occur when varying the cosmological parameters within the $2-\sigma$ current bounds.

As observed in $[12,14]$ spurious peaks dominates the counts at relatively small peak amplitudes while the halo contribution becomes relevant slightly above $s n r^{*} \sim 3$ and dominates at higher values. Since we are mainly interested in how peak counts can constrain the $M-c$ relation, we have to select those peaks associated to real halos. Thus, in the following, we consider only $s n r^{*} \geq 4$ (otherwise, we implicitly assume that spurious contaminations are avoided by means of suitable techniques, see below).

In the previous Section we have shown how the $M_{a p}$ PDF and snr strongly depend on the $M-c$ relation while it is only scarcely affected by variations in the cosmological parameters. Halo concentrations are then anticipated to be crucial in determining the number of halo peaks. Nevertheless, $\sigma_{8}$ and $\Omega_{m}$ are intimately related to the halo mass function, eq. (3.14), and hence expected to largely affect the peak function as well.

We then investigate the impact of halo concentration and cosmological parameters $\sigma_{8}$ and $\Omega_{m}$ on weak lensing peak counts. We do not consider the effects of $H_{0}$ and $n_{s}$ which have only a minor impact on the halo mass function.

Deviations from reference model predictions arising from shifts in normalization $c_{0}$, slope $\alpha$ and scatter $\sigma_{\ln c}$ of $M-c$ relation as well as cosmological parameters $\Omega_{m}$ and $\sigma_{8}$ 


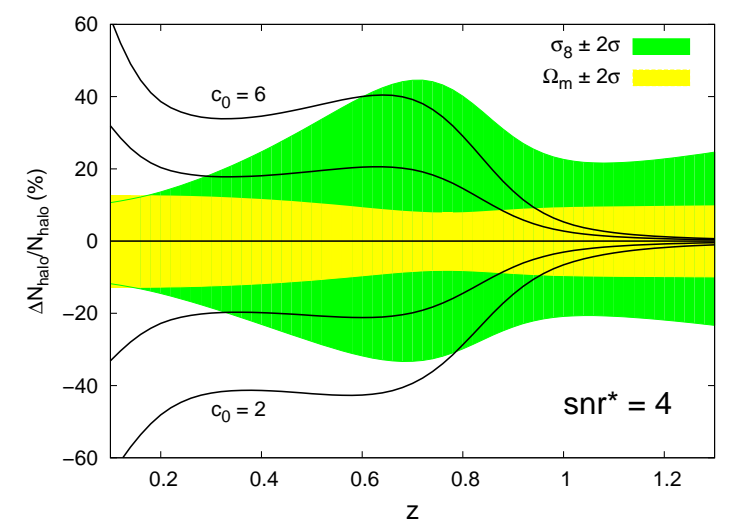

Figure 9. Percentage deviation from the fiducial model peak function due to shifts in $\sigma_{8}, \Omega_{m}$ and $c_{0}$ as in the left panel of fig. 7 .

are considered and compared in Fig. 6. The total number of halo peaks $N_{\text {halo }}\left(>s n r^{*}\right)$ in the redshift range $\Delta z=0.1-1.4$ is shown in the left panels as a function of $s n r^{*}$ while the right ones display the relative deviation, $\Delta N_{\text {halo }} / N_{\text {halo }}=\left[N_{\text {halo }}-N_{\text {halo, fid }}\right] / N_{\text {halo, fid }}$, from the prediction of the fiducial model. Note that, although future galaxy surveys will likely extend over a much greater redshift range, as it will be shown later the number of halo peaks is negligible for $z \gtrsim 0.9$. Shaded regions in figure indicate departures in cosmological parameters (top panels) within the current $2-\sigma$ bounds, and $\pm 25 \%$ offsets in $\alpha$ and $\sigma_{\ln c}$ (bottom panels). Solids lines refers to changes in $c_{0}$.

Among $M-c$ relation parameters, the normalization $c_{0}$ has the major impact on peak counts, yielding deviations from $\sim 25 \%$ to $\sim 75 \%$ at the increasing of $s n r^{*}$ in the range considered, when changing $c_{0}$ of \pm 1 . On the other hand, the effect of varying $\sigma_{\ln c}$ and $\alpha$ is quite modest (or negligible) at low $s n r^{*}$. Nevertheless, the impact of $\sigma_{\ln c}$ rapidly increases at higher $s n r^{*}$.

Observing that the range within which $\Omega_{m}$ and $\sigma_{8}$ are varied correspond to deviations of only $\sim 3-4 \%$, halo counts are certainly more sensitive to cosmological parameters. Within this range, $\sigma_{8}$ yields deviations in halo number of $\sim 25-50 \%$ while they are limited to $\sim 10-15 \%$ when changes in $\Omega_{m}$ are considered.

So far, we considered the total number of halo peaks evaluated from (3.17) by integrating over the full redshift range $\Delta z=0.1-1.4$ thus smoothing out the dependence of the halo mass function (3.14) and the $M_{a p}$ PDF on $z$. Actually, the evolution of the mass function depends on the underlying cosmology through $\sigma_{8}$ and $\Omega_{m}$, while the redshift dependence of the PDF is sensitive to the $M-c$ relation. Thus, it is worth to investigate how peaks are distributed in redshift bins in order to get further insights on the parameters under investigation. To this aim we integrate (3.17) over bins of $\Delta z=0.1$.

Note that, when dealing with real observations, redshift measurements are needed in order to assign a given halo peak to a bin. Redshift information can not be inferred from WL data alone, but additional data are required, e.g. optical data (see the discussion in section 5). On the other hand, spurious peaks are fake detections, not related to a particular halo and not having any real optical counterpart so that no redshift can be attributed to them. Here, we are implicitly and naively assuming that redshifts were properly assigned to halo peaks and spurious detections eliminated from the sample, e.g. by keeping only sufficiently 

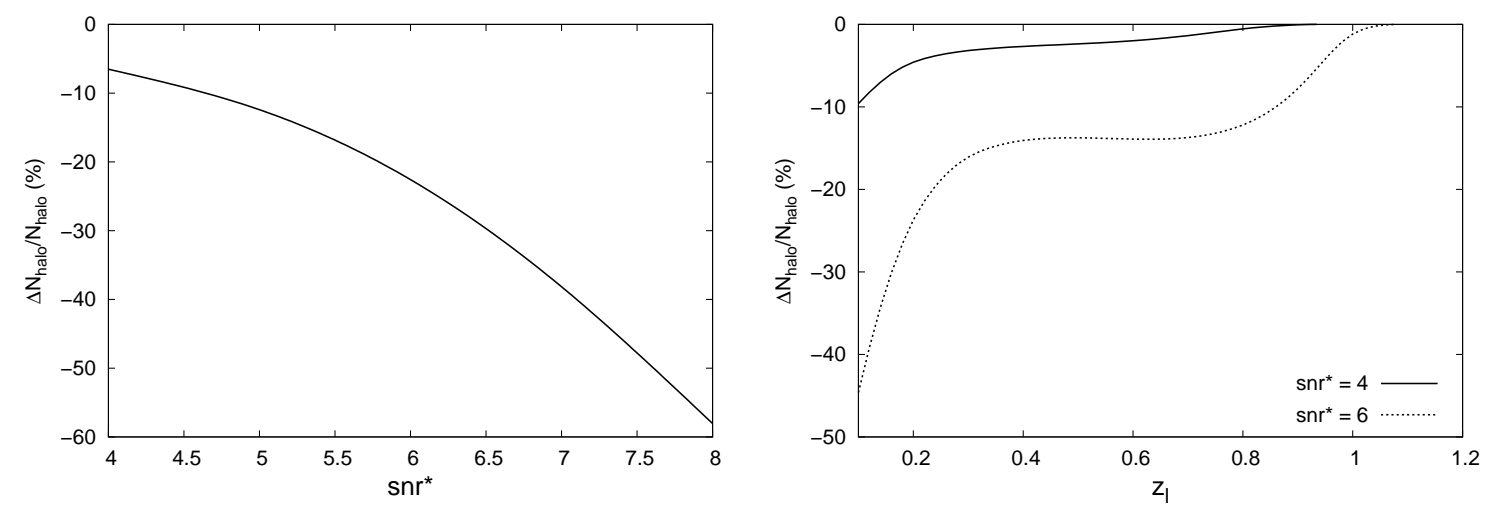

Figure 10. Number counts are shifted toward lower values when concentration scatter is taken into account. The relative shift in $N_{\text {halo }}\left(>s n r^{*}\right)$ and $N_{\text {halo }}\left(>s n r^{*}, z, \Delta z\right)$ from the case when only statistical noise is considered, is shown in the left and right panel respectively.

high peaks and/or by matching WL and optical data (or by means of tomographic techniques, see e.g. [75]).

The resulting peak redshift distribution $N_{\text {halo }}\left(>s n r^{*}, z, \Delta z\right)$ is plotted in figs. 7 and 8 for $s n r^{*}=4$ and 6 respectively in both linear (top panels) and logarithmic (bottom panels) scale in the $\mathrm{y}$-axis to emphasize the behavior at low or high redshifts. Again, deviations from fiducial model predictions due to cosmological (left panels) and $M-c$ relation (right panels) parameters are shown.

Fig. 9 displays the relative deviation, $\Delta N_{\text {halo }} / N_{\text {halo }}$ for the case $s n r^{*}=4$ due to shifts in $c_{0}, \sigma_{8}$ and $\Omega_{m}$. Similar results are obtained for $s n r^{*}=6$ but with larger deviations. Inspection of the figure indicates that probing the high $-z$ tail of the peak function, where it is most sensitive to cosmological parameters, could therefore be an indirect method to investigate the halo mass function.

Finally, we want to show how the number counts change when the concentration scatter is taken into account. As already observed, scatter in $M-c$ relation can affect significantly the $M_{a p}$ variance reducing the $s n r$ for halo peak detections. The net effect is to shift the number counts toward lower values. The relative shift in $N_{\text {halo }}\left(>s n r^{*}\right)$ and $N_{\text {halo }}\left(>s n r^{*}, z, \Delta z\right)$ from the case when only statistical noise is considered, is displayed in the left and right panel, respectively, of fig. 10 .

\section{Fisher matrix forecasts}

In this Section, we employ the Fisher matrix (FM) approach to probe the sensitivities of WL halo number counts to $M-c$ relation and cosmological parameters $\mathbf{p}=\left(c_{0}, \alpha, \sigma_{\ln c}, \sigma_{8}, \Omega_{m}\right)$.

Following [95], the FM for the number of halo peaks $N_{k}\left(z_{k}, \mathbf{p}\right)=N_{\text {halo }}\left(>s n r^{*}, z_{k}, \Delta z\right)$ in the $k$-th redshift bin of width $\Delta z=0.1$ can be written as:

$$
F_{i j}=\sum_{k=1}^{n_{b i n}} \frac{\partial N_{k}}{\partial p_{i}} \frac{\partial N_{k}}{\partial p_{j}} \frac{1}{N_{k}^{f i d}}+\frac{\delta_{i j}}{\sigma_{p}^{2}\left(p_{i}\right)}
$$

where $N_{k}^{f i d}$ is the expected number of peaks in the fiducial model in the $k$-th bin, $n_{b i n}$ is the total number of bins and $\sigma_{p}\left(p_{i}\right)$ is a possible Gaussian prior on the parameter $p_{i}$. The 


\begin{tabular}{|c|c|c|c|c|c|c|}
\hline$s n r^{*}$ & $\sigma\left(c_{0}\right)$ & $\sigma(\alpha)$ & $\sigma\left(\sigma_{\ln c}\right)$ & $\sigma\left(\sigma_{8}\right)$ & $\sigma\left(\Omega_{m}\right)$ & priors \\
\hline 4 & $\begin{array}{c}0.598 \\
(14.9 \%)\end{array}$ & $\begin{array}{c}0.198 \\
(198.2 \%)\end{array}$ & $\begin{array}{c}0.128 \\
(85.3 \%)\end{array}$ & $\begin{array}{c}0.025 \\
(2.9 \%)\end{array}$ & $\begin{array}{c}0.027 \\
(9.2 \%)\end{array}$ & \multirow{2}{*}{ no priors } \\
\hline 6 & $\begin{array}{c}1.535 \\
(38.3 \%)\end{array}$ & $\begin{array}{c}0.976 \\
(975.7 \%)\end{array}$ & $\begin{array}{c}0.096 \\
(64.0 \%)\end{array}$ & $\begin{array}{c}0.178 \\
(21.5 \%)\end{array}$ & $\begin{array}{c}0.181 \\
(62.1 \%)\end{array}$ & \\
\hline 4 & $\begin{array}{c}0.392 \\
(9.8 \%)\end{array}$ & $\begin{array}{c}0.062 \\
(62.5 \%)\end{array}$ & $\begin{array}{c}0.073 \\
(48.6 \%)\end{array}$ & $\begin{array}{c}0.009 \\
(1.0 \%)\end{array}$ & $\begin{array}{c}0.009 \\
(3.0 \%)\end{array}$ & \multirow{2}{*}{$\sigma_{p}\left(\sigma_{8}\right)=\sigma_{p}\left(\Omega_{m}\right)=0.01$} \\
\hline 6 & $\begin{array}{c}0.416 \\
(10.4 \%)\end{array}$ & $\begin{array}{c}0.056 \\
(56.5 \%)\end{array}$ & $\begin{array}{c}0.022 \\
(15.0 \%)\end{array}$ & $\begin{array}{c}0.010 \\
(1.2 \%)\end{array}$ & $\begin{array}{c}0.010 \\
(3.4 \%)\end{array}$ & \\
\hline 4 & $\begin{array}{l}- \\
-\end{array}$ & $\begin{array}{l}- \\
-\end{array}$ & $\begin{array}{l}- \\
-\end{array}$ & $\begin{array}{l}0.0025 \\
(0.3 \%)\end{array}$ & $\begin{array}{l}0.0024 \\
(0.8 \%)\end{array}$ & \multirow{2}{*}{$c_{0}, \alpha, \sigma_{\ln c}$ fixed to fiducial values } \\
\hline 6 & $\begin{array}{l}- \\
-\end{array}$ & $\begin{array}{l}- \\
-\end{array}$ & $\begin{array}{l}- \\
-\end{array}$ & $\begin{array}{l}0.0044 \\
(0.5 \%)\end{array}$ & $\begin{array}{l}0.0064 \\
(2.2 \%)\end{array}$ & \\
\hline
\end{tabular}

Table 2. Fisher matrix forecasted $1-\sigma$ uncertainties on $c_{0}, \alpha, \sigma_{\ln c}, \sigma_{8}$ and $\Omega_{m}$. Results are obtained for $s n r^{*}=4$ and 6 assuming: i) no priors, ii) a prior of 0.01 on both $\sigma\left(\sigma_{8}\right)$ and $\sigma\left(\Omega_{m}\right)$, iii) a perfect knowledge of $M-c$ relation. The percentages in parentheses denote the relative error $\sigma\left(p_{i}\right) / p_{i}$.

\begin{tabular}{|c|rrrrr|}
\hline$s n r^{*}=4$ & $c_{0}$ & $\alpha$ & $\sigma_{\ln c}$ & $\sigma_{8}$ & $\Omega_{m}$ \\
\hline$\Omega_{m}$ & -0.52 & 0.93 & -0.84 & -0.81 & 1.00 \\
$\sigma_{8}$ & -0.07 & -0.94 & 0.42 & 1.00 & \\
$\sigma_{\ln c}$ & 0.85 & -0.69 & 1.00 & & \\
$\alpha$ & -0.24 & 1.00 & & & \\
$c_{0}$ & 1.00 & & & & \\
\hline
\end{tabular}

\begin{tabular}{|c|rrrrr|}
\hline$s n r^{*}=6$ & $c_{0}$ & $\alpha$ & $\sigma_{\ln c}$ & $\sigma_{8}$ & $\Omega_{m}$ \\
\hline$\Omega_{m}$ & -0.69 & 0.99 & 0.68 & -0.97 & 1.00 \\
$\sigma_{8}$ & 0.49 & -0.99 & -0.84 & 1.00 & \\
$\sigma_{\ln c}$ & 0.06 & 0.75 & 1.00 & & \\
$\alpha$ & -0.61 & 1.00 & & & \\
$c_{0}$ & 1.00 & & & & \\
\hline
\end{tabular}

Table 3. Correlation coefficients $\rho_{i j}=\sigma_{i j} / \sigma\left(p_{i}\right) \sigma\left(p_{j}\right)$ for $s n r^{*}=4$ (top) and $s n r^{*}=4$ (bottom).

\begin{tabular}{|cccc|}
\hline$s n r^{*}$ & $M_{\text {piv }} / 10^{14} h^{-1} M_{\odot}$ & $c_{\text {piv }}$ & $\sigma_{c_{\text {piv }}}$ \\
\hline 4 & 1.19 & 3.93 & $0.57(14.5 \%)$ \\
6 & 1.27 & 3.91 & $1.19(30.5 \%)$ \\
\hline
\end{tabular}

Table 4. $M_{\text {piv }}, c_{\text {piv }}$, and $\sigma_{c_{\text {piv }}}$ for $s n r^{*}=4$ and 6.

inverse of FM then gives the covariance matrix $C_{i j}$, the diagonal elements of which represent the lowest variance $\sigma\left(p_{i}\right)$ one can achieve on the parameter $p_{i}$.

The forecasted uncertainties $\sigma\left(p_{i}\right)$ turn out to depend on the threshold $s n r^{*}$ and are summarized in table 2 together with the percentage errors $\sigma\left(p_{i}\right) / p_{i}$ (in parentheses) for $s n r^{*}=$ 4 and 6. In addition, the correlation coefficients $\rho_{i j}=\sigma_{i j} / \sigma\left(p_{i}\right) \sigma\left(p_{j}\right)\left(\sigma_{i j}\right.$ being the covariance between $p_{i}$ and $p_{j}$ ) are shown in table 3 . 


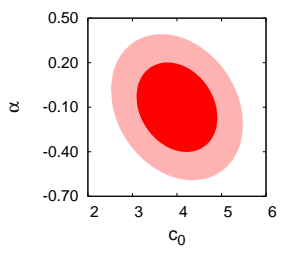

$$
\mathrm{snr}^{*}=4
$$
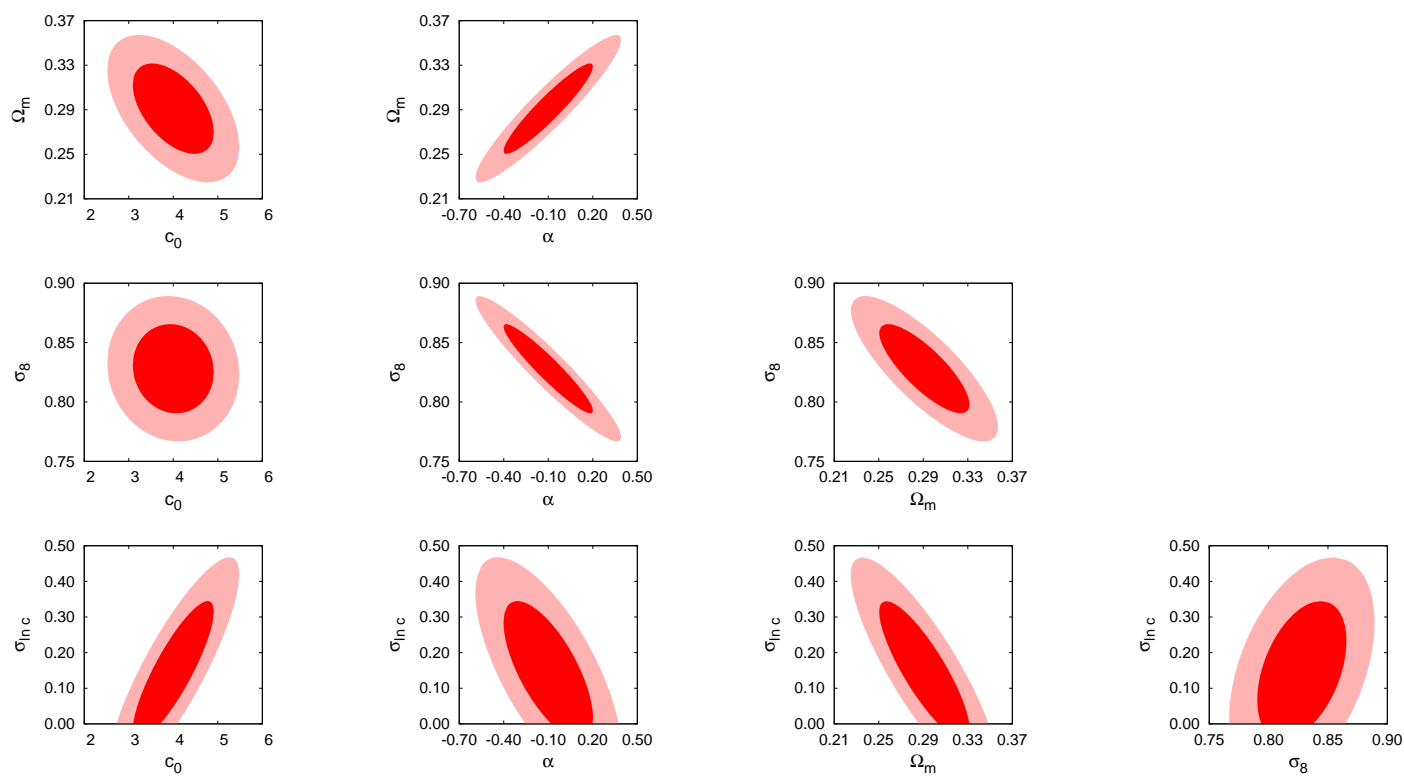

Figure 11. 1- and $2-\sigma$ marginalized Fisher ellipses for $c_{0}, \alpha, \sigma_{\ln c}, \sigma_{8}$ and $\Omega_{m}$ in the case $s n r^{*}=4$.

We however warn the reader that the FM forecasts given in table 2, should be taken as optimistic limits (see the discussion in the next Section).

In the absence of any prior information, we first note how peak statistics turns out to be competitive with other cosmological probes in placing constraints on $\sigma_{8}$ and $\Omega_{m}$. For $s n r^{*}=4$ we indeed obtain $\sigma\left(\sigma_{8}\right)=0.025$ and $\sigma\left(\Omega_{m}\right)=0.027$ which are very close to the current limits from Cosmic Microwave Background (CMB) or LSS data.

Turning to $M-c$ relation, the most constrained parameter is the normalization $c_{0}$. For $s n r^{*}=4$, we find $\sigma\left(c_{0}\right) / c_{0} \sim 15 \%$, which, at first glance, could look a not so encouraging result. We stress, however, that this is still an appreciable result, considered the wide variety of $c_{0}$ values reported in literature. On the other hand, peak statistics alone seems less efficient in putting limits on $\sigma_{\ln c}$ and in particular on the slope $\alpha$ which is substantially unconstrained. Going to $s n r^{*}=6$ has the effect of reducing the overall number of peaks so that a worsening of the constraints is expected because of the poorer statistics. This is not, however, the case for $\sigma_{\ln c}$. This is likely related to the fact that larger $s n r$ probe larger masses which are more sensitive to $\sigma_{\ln c}$ especially at moderate low redshifts where the number of halo peaks is still significative (compare figs. 7 and 8).

In light of these results, peak statistics alone seems not to be helpful in constraining the $M-c$ relation. We then consider the effect of combining peak counts with current/near-future observations in order to probe whether the constraints can be improved.

Current data from CMB, LSS and type Ia Supernovae already constrain $\Omega_{m}$ with an uncertainty $\sigma\left(\Omega_{m}\right) \sim 0.01$ while set the most stringent bounds on $\sigma_{8}$ to $\sigma\left(\sigma_{8}\right) \sim 0.015-0.018$, 

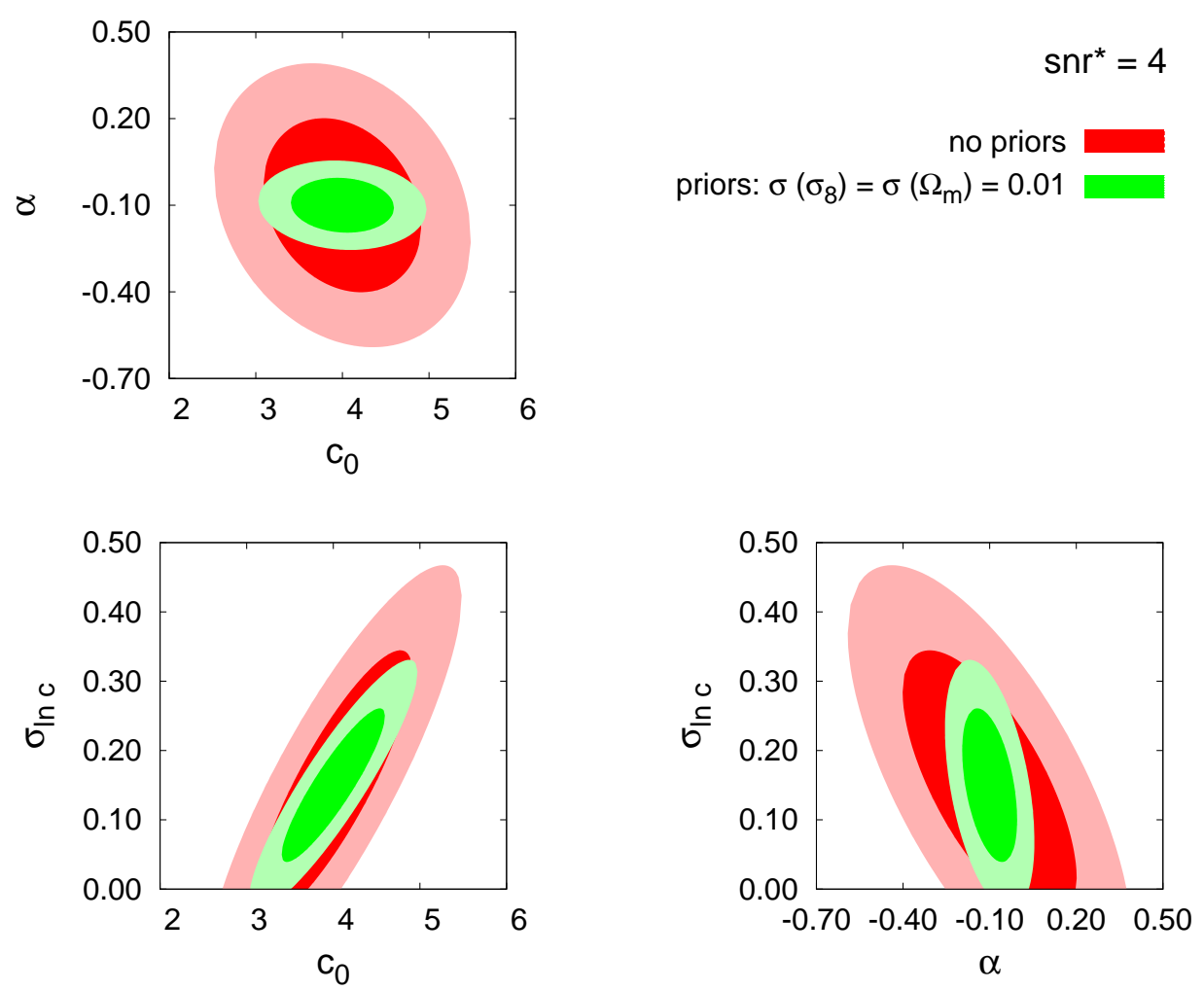

Figure 12. $1-$ and $2-\sigma$ constraints on the $M-c$ relation parameters $\left(c_{0}, \alpha, \sigma_{\ln c}\right)$ obtained for $s n r^{*}=4$ assuming: i) no priors, ii) a prior of 0.01 on both $\sigma\left(\sigma_{8}\right)$ and $\sigma\left(\Omega_{m}\right)$.

depending on the dataset used (see e.g [86, 96]; see also [97] for an analysis based on WL tomography). Nevertheless, assuming an uncertainty of $\sigma\left(\sigma_{8}\right)=0.01$ is not unreasonable. It is just less than half of the current limit and, hopefully, it will be achieved by a joint analysis of current and/or near future cosmological probes (see, however, [98] where cluster mass function measurements are used in combination with other dataset to give $\sigma\left(\sigma_{8}\right) \sim 0.01$ ).

We thus include Gaussian priors $\sigma_{p}\left(\sigma_{8}\right)=\sigma_{p}\left(\Omega_{m}\right)=0.01$ in the analysis finding a significant improvement in the constraints on $M-c$ relation. Uncertainties on $c_{0}$ reduce to $\sim 10 \%$ for both $s n r=4$ and $s n r=6$ while $\sigma_{\ln c}$ is reasonably (marginally) constrained for $s n r=6$ (4). Despite the large confidence interval, constraints on $\alpha$ are however competitive with those from strong lensing and X-ray observations (se e.g. [37, 51, 54, 99]).

In fig. 11 we display the 2D $68 \%$ and $95 \%$ marginalized confidence regions for different pairs of parameters for $s n r^{*}=4$ and no priors added. Fig. 12 shows how the Fisher ellipses for $c_{0}, \alpha$ and $\sigma_{\ln c}$ are reduced after placing the priors $\sigma_{p}\left(\sigma_{8}\right)=\sigma_{p}\left(\Omega_{m}\right)=0.01$. Finally, the $2 \mathrm{D}$ constraints for $s n r^{*}=4$ and $s n r^{*}=6$ are compared in fig. 13 .

On the other hand, it is also interesting to investigate the power of the WL peak statistics to constrain the cosmological parameters when one or more $M-c$ relation parameters are known with good accuracy. To this aim, we consider the following cases where a prior $\sigma_{p}$ is added on: i) $c_{0}$, ii) $\alpha$, iii) $\sigma_{\ln c}$, iv) all the $M-c$ relation parameters. Fig. 14 shows the uncertainties on $\Omega_{m}$ and $\sigma_{8}$ at the varying of $\sigma_{p}$ for the cases $s n r^{*}=4$ and 6 . Results can be understood inspecting the correlation coefficients listed in table 3. Among the $M-c$ 


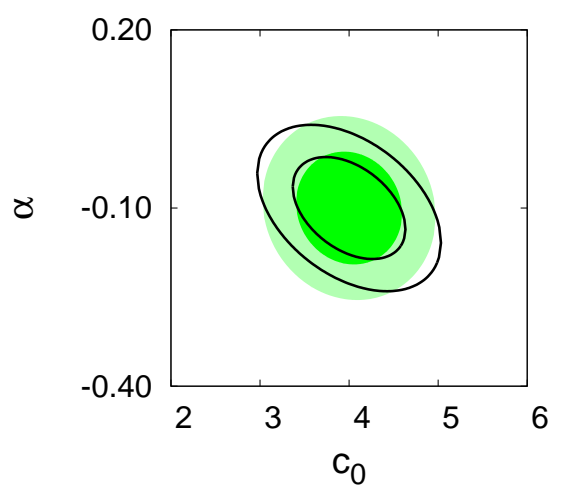

priors: $\sigma\left(\sigma_{8}\right)=\sigma\left(\Omega_{\mathrm{m}}\right)=0.01$
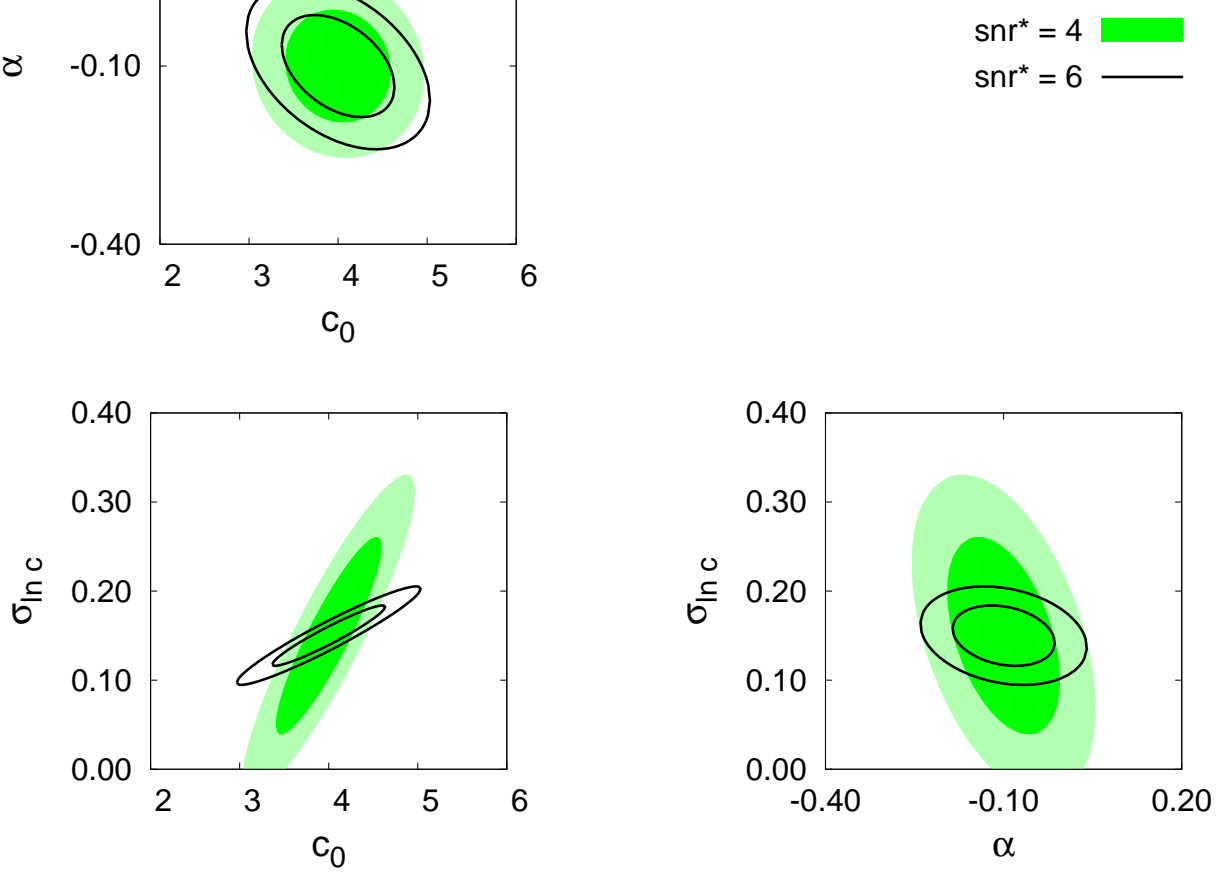

Figure 13. $1-$ and $2-\sigma$ constraints on the $M-c$ relation parameters $\left(c_{0}, \alpha, \sigma_{\ln c}\right)$. Results are obtained for $s n r^{*}=4$ and 6 placing a prior of 0.01 on both $\sigma\left(\sigma_{8}\right)$ and $\sigma\left(\Omega_{m}\right)$.

relation parameters, the slope $\alpha$ is completely (anti)correlated with $\left(\sigma_{8}\right) \Omega_{m}$ so that it just suffices to know $\alpha$ with a sufficient precision to significantly reduce the cosmological parameter uncertainties. For our fiducial model and $s n r^{*}=4$, constraints on $\sigma_{8}$ and $\Omega_{m}$ improve of about $\sim 50 \%(\sim 70 \%)$ (with respect to the values reported in the first row of table 2) if $\alpha$ is known with an uncertainty of $\sigma(\alpha) / \alpha \simeq 1(<0.1)$. Similar values are obtained for $s n r^{*}=6$. On the contrary, given their lower correlations, even with a perfect knowledge of $c_{0}$ or $\sigma_{\ln c}$ limits on cosmological parameters are only modestly/scarcely reduced. The last two rows of table 2 show the uncertainties on $\Omega_{m}$ and $\sigma_{8}$ in the ideal case in which the $M-c$ relation is perfectly known. Although these numbers are too good and too optimistic and can not happen for real measurements, the above discussion highlights the importance of studies of the halo concentration parameters (in particular the slope $\alpha$ ) for future surveys.

Finally, it is reasonable to suppose that, the $M_{a p}$ statistics can probe the inner structure of halos only for sufficiently massive halos. It is then interesting to investigate which mass scale can better constrain the concentration parameter. To this aim we follow [100] (see also $[101,102]$ for the same approach in a different contest) and define a pivot mass $M_{\text {piv }}$ for the 

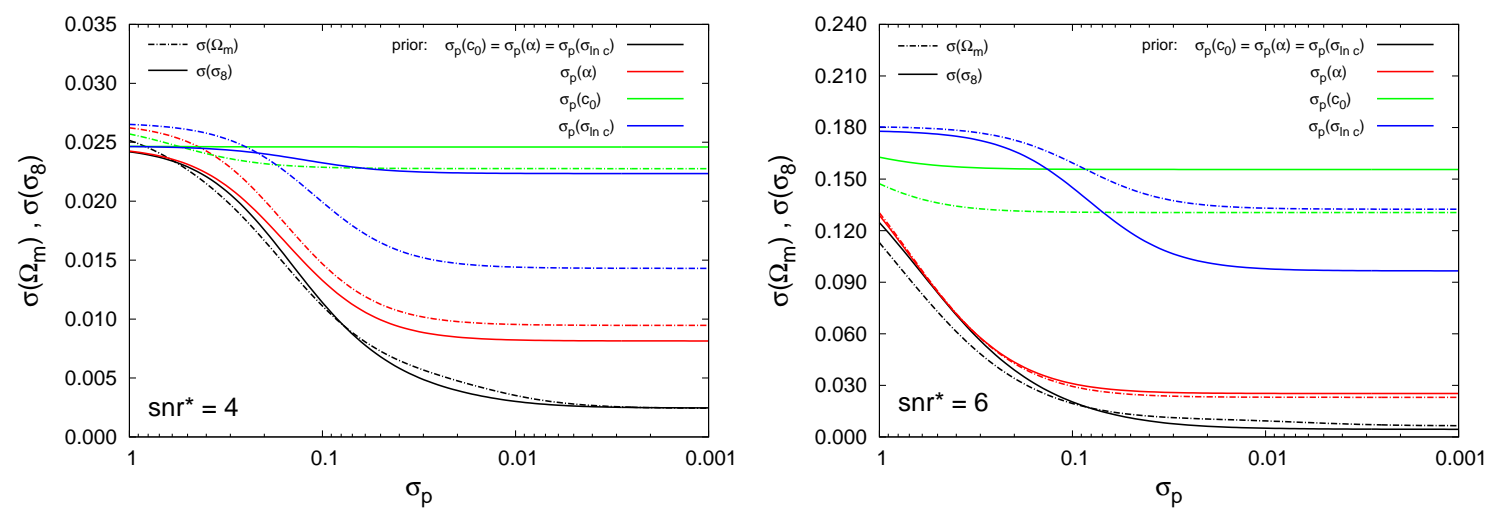

Figure 14. Fisher matrix forecasted $1-\sigma$ uncertainties on $\sigma_{8}$ and $\Omega_{m}$ at the varying of the priors on $M-c$ relation parameters. Results are given for $s n r^{*}=4$ (left panel) and 6 (right panel).

$M-c$ relation. We then choose $M_{\text {piv }}$ such that the relative error:

$$
\begin{aligned}
\frac{\sigma_{c_{\mathrm{piv}}}^{2}}{c_{\mathrm{piv}}^{2}} & =\frac{1}{c_{\mathrm{piv}}^{2}} \sum_{i, j} \frac{\partial c_{\mathrm{piv}}}{\partial p_{i}} C_{i j} \frac{\partial c_{\mathrm{piv}}}{\partial p_{j}} \\
& =\frac{C_{c_{0} c_{0}}}{c_{0}^{2}}+\left[\ln \left(\frac{M_{\mathrm{piv}}}{M_{0}}\right)\right]^{2} C_{\alpha \alpha}+\frac{2}{c_{0}} \ln \left(\frac{M_{\mathrm{piv}}}{M_{0}}\right) C_{c_{0} \alpha}
\end{aligned}
$$

(here $C_{i j}=\left(F^{-1}\right)_{i j}$ is the parameter covariance matrix and $M_{0}=10^{14} h^{-1} M_{\odot}$, see (2.9)) on the rescaled normalization $c_{\text {piv }}=c_{0}\left(M_{\text {piv }} / M_{0}\right)^{\alpha}$ is minimized (i.e. the errors on $c_{\text {piv }}$ and $\alpha$ are uncorrelated). This occurs when

$$
\ln \left(\frac{M_{\mathrm{piv}}}{M_{0}}\right)=-\frac{1}{c_{0}} \frac{C_{c_{0} \alpha}}{C_{\alpha \alpha}}
$$

yielding

$$
\frac{\sigma_{c_{\mathrm{piv}}}^{2}}{c_{\mathrm{piv}}^{2}}=\frac{1}{c_{0}^{2}}\left[C_{c_{0} c_{0}}-\frac{C_{c_{0} \alpha}^{2}}{C_{\alpha \alpha}}\right]
$$

The values of $M_{\text {piv }}, c_{\text {piv }}$ and $\sigma_{\text {piv }}$ as a function of $s n r^{*}$ are displayed in fig. 15 . As the figure shows, $M_{\text {piv }}$ is quite insensitive to the peak height threshold up to $s n r^{*} \simeq 7$, ranging from $\sim 1.2$ to $\sim 1.5 \cdot 10^{14} h^{-1} M_{\odot}$. Then, it rapidly increases up to $\sim 3.5 \cdot 10^{14} h^{-1} M_{\odot}$ at $s n r^{*}=8$. This is probably due to the fact that, for such high thresholds, the signal is mostly due to more massive halos which, unlike the less massive ones, feel the impact of the concentration scatter (see section 3.1). Results for $s n r^{*}=4$ and 6 are very close to those reported in table 2 (first two rows) and are summarized in table 4.

It is worth mentioning, that peak number counts depend on the adopted filter function so that the constraints here reported can change if a different filter is used. Investigating which filter is optimal for peaks detection is outside the aims of this work. We only mention that a filter which performs better than the one used here, would increase the number of detections improving the counts statistics and consequently the constraints reported in table 2 . 


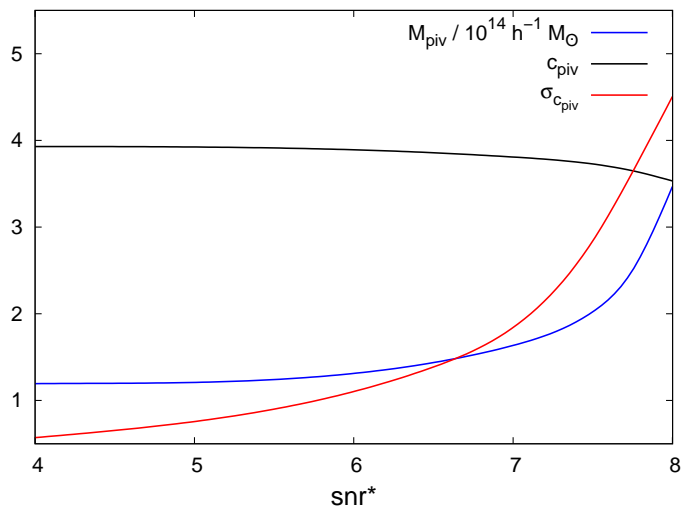

Figure 15. $M_{\text {piv }}, c_{\text {piv }}$, and $\sigma_{c_{\text {piv }}}$ as a function of $s n r^{*}$ (see text for details).

\section{Discussion and conclusions}

Halo masses and concentrations have been studied extensively, by means of N-body simulations as well as observationally, during the last decade. Nevertheless, the exact form of the $M-c$ relation is still widely debated. One of the most promising method to estimate masses and concentrations relies on gravitational lensing, in particular shear peak counts from nearfuture WL surveys seem a promising and complementary method to delineate the features of the $M-c$ relation, as pointed out in this work.

The method for shear peak detection we have considered here relies on the $M_{a p}$ statistics. Hence, we have firstly investigated the impact of the $M-c$ relation on the PDF of $M_{a p}$. In particular, we have provided a prescription to properly take into account the $M-c$ relation dispersion, always disregarded in previous work, in the PDF. We have then shown that such scatter can affect significantly the PDF and, consequently, the snr for peak detection.

Secondly, we have investigated the impact of the $M-c$ relation on peaks number counts as compared to that of the cosmological parameters $\sigma_{8}$ and $\Omega_{m}$.

Finally, we have performed a Fisher matrix analysis in order to assess the capability of an Euclid-like survey in constraining the $M-c$ relation and cosmological parameters by using peak number counts. We have found that: i) peak function alone provides constraints on $\sigma_{8}$ and $\Omega_{m}$ which are competitive with those obtained from different probes. On the other hand, only the normalization $c_{0}$ of the $M-c$ relation is reasonably (but not so strongly) constrained if peaks with $s n r>4$ are considered; ii) adding prior informations on $\sigma_{8}$ and $\Omega_{m}$ as inferred from current/near-future data, constraints on $M-c$ relation improve significantly so that peak statistics seems helpful in discriminating among the wide variety of $M-c$ relation fits found in literature although no substantial new information on the slope $\alpha$ is inferred.

Although results are quite encouraging, it is worth stressing that the Fisher matrix analysis here presented disregards the effects of the halo sample variance on the error estimates, only Poisson noise being considered. Nevertheless, sample variance might be significant for low mass halos at low redshifts where their abundance is much higher thus reducing the Poisson noise. To yield more accurate predictions, the covariance matrix for number counts $C_{i j}{ }^{9}$, entering the FM expression, should then be written as: $C_{i j}=P_{i j}+S_{i j}$ where $P_{i j}=\delta_{i j} N_{i}^{f i d}$

\footnotetext{
${ }^{9}$ it should not be confused with the parameter covariance matrix of eq. (4.2) for which we use the same simbol.
} 
and $S_{i j}=N_{i}^{f i d} N_{j}^{f i d} b_{i} b_{j} \sigma_{i j}^{D M}$ are the Poissonian and sample variance contributions respectively (here $N_{i}^{f i d}$ and $b_{i}$ are the expected number of peaks and the mean halo bias in the $i$-th redshift bin, while $\sigma_{i j}^{D M}$ is the covariance of the dark matter fluctuations between the bins $i$ and $j$, se e.g. [103-105] for more details).

Sample variance effects on halo number counts have been considered in previous works, e.g. [103-107]. In particular, [103, 105] conclude that sample variance is generally comparable or greater than Poisson noise for number counts above mass threshold $M_{t h} \lesssim 1-4 \cdot 10^{14} h^{-1} M_{\odot}$ depending on the survey width and depth. In our case, the mass thresholds corresponding to given $s n r^{*}$ and $z$ can be deduced from the middle panel of fig. 4. According to $[103,105]$, for $s n r^{*} \geq 4$ we expect sample variance to have only moderate effects in the redshift range $\Delta z=0.15-0.45$ where the counts signal is maximum, becoming negligible at the increasing of $z$. For a raw estimate, we can assume $C_{i j}$ to be diagonal (i.e. halo correlation length smaller than the bin width) and $S_{i j}=P_{i j}\left(S_{i j}=2 P_{i j}\right)$ independently of the redshift. This yields a worsening of the parameter constraints by a factor $\sqrt{2}(\sqrt{3})$.

However, [103-105] neglect the information arising from the cosmological dependence of the sample variance. When this information is included and considered as "signal", it could cause a smaller degradation in the constraints or, in case, even improvements $[106,107]$. In this case, the full FM can be well approximated as:

$$
F_{i j}=\mathbf{N}_{, i}^{T} \mathbf{C}^{-1} \mathbf{N}_{, j}+\frac{1}{2} \operatorname{Tr}\left[\mathbf{C}^{-1} \mathbf{S}_{, i} \mathbf{C}^{-1} \mathbf{S}_{, j}\right]+\frac{\delta_{i j}}{\sigma_{p}^{2}\left(p_{i}\right)}
$$

where $\mathbf{N}=\left(N_{1}^{f i d}, \ldots, N_{n_{b i n}}^{f i d}\right)$ and ${ }_{, i}$ denotes the derivative with respect to the parameter $p_{i}$. It is worth stressing that the second term, neglected in [103-105], beside the information about the cosmological dependence of the sample variance, also contains information about its dependence on $M-c$ relation trough the derivative of $N_{i}^{f i d}$ and possibly of $b_{i}$. Indeed, it was pointed out in $[108,109]$ that halo bias could depend not only on the halo mass but also on additional halo properties, among which the concentration.

Adding sample variance information could then, in principle, provide an opportunity to improve measurements of halo concentration (and cosmological parameters) with WL peak statistics through self-calibration techniques for $M-c$ relation similar to those for massobservable relation (see e.g. $[106,107])$. This intriguing point, which deserve a detailed analysis, is left for future works.

A second issue concerns the assumption that detected peaks can be properly assigned to redshift bins. The redshift information, however, can not be extracted from WL data only, but additional data need to be included. In order to split the peak sample in redshift bins, optical finders (see e.g. [110-113]) could serve the purpose since they return reasonable redshift estimates, especially because they can be applied on the same optical data retrieved for lensing. Although the correlation between optical detections and WL peaks is not trivial, in a Euclid-like survey, one can look at the position of the peaks in the optical images, and then deduce the peak redshift $z$ from the redshifts of the galaxies closest to the peak position. In a first approximation, we can suppose that this method provides a Gaussian probability distribution function for $z$ with negligible bias and variance $\sigma_{z}=\sigma_{0}(1+z)$, and then assume a peak to be correctly assigned to a redshift bin roughly asking that the $3-\sigma$ uncertainty on $z$ is smaller than the bin width.

For our assumed value $\Delta z=0.1$, this translates in $\sigma_{0} \leq 0.03$, a precision which could be likely achieved if $z$ is spectroscopically measured $\left(\sigma_{0} \simeq 0.001\right.$ according to the Euclid red 
book), but could be too demanding if one relies on photometric redshift methods $\left(\sigma_{0} \simeq 0.05\right.$ for Euclid). In this second case, one should add a non Poissonian uncertainty on $N_{\text {halo }}(s n r>$ $\left.s n r^{*}, z, \Delta z\right)$ in the above Fisher matrix analysis and resort to the pull statistics [114]. On the other hand, it is likely that the precision of the inferred redshift also depends on the snr of peak so that the net effect should be included in the analysis by convolving the theoretically computed $N_{\text {halo }}$ with an empirically determined selection function.

A further issue concerns the assumption of NFW spherical halos. Real halos are not spherical and not all relaxed so that deviations from the universal NFW profile are expected. The effects of the diversity of dark matter distributions in individual halos on peak counts has been investigated in $[80,82]$ by means of numerical simulations and analytic methods. The noise originated from halo shape is found to be comparable to the statistical noise discussed in section 3.9. Furthermore, halo orientations cause a systematic bias in the peak heights. In order to include these effects in the theoretical predictions, one should consider a triaxial halo model (see [85]) which is shown to work quite well [82].

It is also worth mentioning that, in the FM analysis, we have only investigated a subset of the full parameter space. Here, we were mainly interested in how peak statistics constrain the $M-c$ relation so that those parameters, as $H_{0}$ and $n_{s}$, which have a minor impact on peak number counts and are expected to be scarcely constrained, were held fixed to their fiducial values. Nevertheless, when dealing with real data, the full parameter space should be considered. Allowing for a large number of parameters to be varied introduces further degeneracies widening the confidence regions. This degradation in the constraining power, can then be compensated by complementing peak statistics with further datasets, e.g. SNeIa and CMB.

Investigating the above issue is however outside our aims here.

\section{Acknowledgments}

$\mathrm{RM}$ is grateful to S. Bonometto for carefully reading the manuscript, helpful comments and suggestions. G. La Vacca is also thanked for useful discussions.

After this paper has been completed, we became aware of a very similar work by V. Cardone, S. Camera, M. Sereno, G. Covone, R. Maoli and R. Scaramella [115]. We thank them for sharing their draft paper and useful discussions.

\section{References}

[1] M. Hetterscheidt, P. Simon, M. Schirmer, H. Hildebrandt, T. Schrabback, T. Erben, and P. Schneider, GaBoDS: The Garching-Bonn deep survey. VII. Cosmic shear analysis, A\&A 468 (2007) 859-876, [astro-ph/0606571].

[2] H. Hoekstra, Y. Mellier, L. van Waerbeke, E. Semboloni, L. Fu, M. J. Hudson, L. C. Parker, I. Tereno, and K. Benabed, First Cosmic Shear Results from the Canada-France-Hawaii Telescope Wide Synoptic Legacy Survey, ApJ 647 (2006) 116-127, [astro-ph/0511089].

[3] E. Semboloni, Y. Mellier, L. van Waerbeke, H. Hoekstra, I. Tereno, K. Benabed, S. D. J. Gwyn, L. Fu, M. J. Hudson, R. Maoli, and L. C. Parker, Cosmic shear analysis with CFHTLS deep data, A\&A 452 (2006) 51-61, [astro-ph/0511090].

[4] R. Laurejis et al., Euclid Definition Study Report, (2011), [arXiv:1110.3193].

[5] L. Amendola et al., Cosmology and Fundamental Physics with the Euclid Satellite, Living Reviews in Relativity 16 (2013), [arXiv: 1206.1225]. 
[6] G. Kruse and P. Schneider, Statistics of dark matter haloes expected from weak lensing surveys, MNRAS 302 (1999) 821-829, [astro-ph/9806071].

[7] K. Reblinsky, G. Kruse, B. Jain, and P. Schneider, Cosmic shear and halo abundances: analytical versus numerical results, A\&A 351 (1999) 815-826, [astro-ph/9907250].

[8] J. P. Dietrich and J. Hartlap, Cosmology with the shear-peak statistics, MNRAS 402 (2010) 1049-1058, [arXiv: 0906.3512].

[9] H. Hoekstra, The effect of distant large scale structure on weak lensing mass estimates, A\&A 370 (2001) 743-753, [astro-ph/0102368].

[10] H. Hoekstra, How well can we determine cluster mass profiles from weak lensing?, MNRAS 339 (2003) 1155-1162, [astro-ph/0208351].

[11] H. Hoekstra, J. Hartlap, S. Hilbert, and E. van Uitert, Effects of distant large-scale structure on the precision of weak lensing mass measurements, MNRAS 412 (2011) 2095-2103, [arXiv: 1011.1084$]$.

[12] T. Hamana, M. Takada, and N. Yoshida, Searching for massive clusters in weak lensing surveys, MNRAS 350 (May, 2004) 893-913, [astro-ph/0310607].

[13] L. Marian and G. M. Bernstein, Dark energy constraints from lensing-detected galaxy clusters, Phys. Rev. D 73 (2006), no. 12 123525, [astro-ph/0605746].

[14] M. Maturi, C. Angrick, F. Pace, and M. Bartelmann, An analytic approach to number counts of weak-lensing peak detections, A\&A 519 (2010) A23, [arXiv:0907.1849].

[15] M. Maturi, C. Fedeli, L. Moscardini, Imprints of primordial non-Gaussianity on the number counts of cosmic shear peaks, MNRAS 416 (2011) 2527-2538, [arXiv:1101.4175].

[16] V. F. Cardone, S. Camera, R. Mainini, A. Romano, A. Diaferio, R. Maoli, R. Scaramella, Weak lensing peak count as a probe of $f(R)$ theories, MNRAS 430 (2013) 2896-2909, [arXiv: 1204.3148].

[17] M. Hetterscheidt, T. Erben, P. Schneider, R. Maoli, L. van Waerbeke, and Y. Mellier, Searching for galaxy clusters using the aperture mass statistics in 50 VLT fields, A\&A 442 (2005) 43-61, [astro-ph/0504635].

[18] H. Dahle, The Cluster Mass Function from Weak Gravitational Lensing, ApJ 653 (2006) 954-962, [astro-ph/0608480].

[19] D. Wittman, I. P. Dell'Antonio, J. P. Hughes, V. E. Margoniner, J. A. Tyson, J. G. Cohen, and D. Norman, First Results on Shear-selected Clusters from the Deep Lens Survey: Optical Imaging, Spectroscopy, and X-Ray Follow-up, ApJ 643 (2006) 128-143, [astro-ph/0507606].

[20] R. Gavazzi and G. Soucail, Weak lensing survey of galaxy clusters in the CFHTLS Deep, A\&A 462 (2007) 459-471, [astro-ph/0605591].

[21] M. Schirmer, T. Erben, M. Hetterscheidt, and P. Schneider, GaBoDS: the Garching-Bonn Deep Survey. IX. A sample of 158 shear-selected mass concentration candidates, A\&A 462 (2007) 875-887, [astro-ph/0607022].

[22] S. Miyazaki, T. Hamana, R. S. Ellis, N. Kashikawa, R. J. Massey, J. Taylor, and A. Refregier, A Subaru Weak-Lensing Survey. I. Cluster Candidates and Spectroscopic Verification, ApJ 669 (2007) 714-728, [arXiv:0707.2249].

[23] J. Bergé, F. Pacaud, A. Réfrégier, R. Massey, M. Pierre, A. Amara, M. Birkinshaw, S. Paulin-Henriksson, G. P. Smith, and J. Willis, Combined analysis of weak lensing and X-ray blind surveys, MNRAS 385 (2008) 695-707, [arXiv: 0712.3293].

[24] A. Abate, D. Wittman, V. E. Margoniner, S. L. Bridle, P. Gee, J. A. Tyson, and I. P. Dell'Antonio, Shear-selected Clusters from the Deep Lens Survey. III. Masses from Weak Lensing, ApJ 702 (2009) 603-613, [arXiv:0904.2185]. 
[25] H. Shan, J.-P. Kneib, C. Tao, Z. Fan, M. Jauzac, M. Limousin, R. Massey, J. Rhodes, K. Thanjavur, and H. J. McCracken, Weak lensing measurement of galaxy clusters in the CFHTLS-Wide survey, (2011) [arXiv:1108.1981].

[26] N. Okabe, Y.-Y. Zhang, A. Finoguenov, M. Takada, G. P. Smith, K. Umetsu, and T. Futamase, LoCuSS: Calibrating Mass-observable Scaling Relations for Cluster Cosmology with Subaru Weak-lensing Observations, ApJ 721 (2010) 875-885, [arXiv:1007.3816].

[27] H. Israel, T. Erben, T. H. Reiprich, A. Vikhlinin, H. Hildebrandt, D. S. Hudson, B. A. McLeod, C. L. Sarazin, P. Schneider, and Y.-Y. Zhang, The 400d Galaxy Cluster Survey weak lensing programme. I. MMT/Megacam analysis of CL0030+2618 at $z=0.50$, A\&A 520 (2010) A58, [arXiv:0911.3111].

[28] P. Schneider, Detection of (dark) matter concentrations via weak gravitational lensing, MNRAS 283 (1996) 837-853, [astro-ph/9601039].

[29] L. J. King and J. M. G. Mead, The mass-concentration relationship of virialized haloes and its impact on cosmological observables, MNRAS 416 (2011) 2539-2549, [arXiv:1105.3155].

[30] J. S. Bullock, T. S. Kolatt, Y. Sigad, R. S. Somerville, A. V. Kravtsov, A. A. Klypin, J. R. Primack, and A. Dekel, Profiles of dark haloes: evolution, scatter and environment, MNRAS 321 (2001) 559-575, [astro-ph/9908159].

[31] V. R. Eke, J. F. Navarro, and M. Steinmetz, The Power Spectrum Dependence of Dark Matter Halo Concentrations, ApJ 554 (2001) 114-125, [astro-ph/0012337].

[32] J. M. Comerford and P. Natarajan, The observed concentration-mass relation for galaxy clusters, MNRAS 379 (July, 2007) 190-200, [astro-ph/0703126].

[33] A. F. Neto, L. Gao, P. Bett, S. Cole, J. F. Navarro, C. S. Frenk, S. D. M. White, V. Springel, and A. Jenkins, The statistics of $\Lambda$ CDM halo concentrations, MNRAS 381 (2007) 1450-1462, [arXiv:0706.2919].

[34] L. Gao, J. F. Navarro, S. Cole, C. S. Frenk, S. D. M. White, V. Springel, A. Jenkins, and A. F. Neto, The redshift dependence of the structure of massive $\Lambda$ cold dark matter haloes, MNRAS 387 (2008) 536-544, [arXiv:0711.0746].

[35] A. R. Duffy, J. Schaye, S. T. Kay, and C. Dalla Vecchia, Dark matter halo concentrations in the Wilkinson Microwave Anisotropy Probe year 5 cosmology, MNRAS 390 (2008) L64-L68, [arXiv:0804.2486].

[36] A. V. Macciò, A. A. Dutton, and F. C. van den Bosch, Concentration, spin and shape of dark matter haloes as a function of the cosmological model: WMAP1, WMAP3 and WMAP5 results, MNRAS 391 (2008) 1940-1954, [arXiv:0805.1926].

[37] R. Mandelbaum, U. Seljak, and C. M. Hirata, A halo mass-concentration relation from weak lensing, J. Cosmology Astropart. Phys. 8 (2008) 6, [arXiv:0805.2552].

[38] M. Oguri, J. F. Hennawi, M. D. Gladders, H. Dahle, P. Natarajan, N. Dalal, B. P. Koester, K. Sharon, and M. Bayliss, Subaru Weak Lensing Measurements of Four Strong Lensing Clusters: Are Lensing Clusters Overconcentrated?, ApJ 699 (2009) 1038-1052, [arXiv: 0901.4372].

[39] A. A. Klypin, S. Trujillo-Gomez, and J. Primack, Dark Matter Halos in the Standard Cosmological Model: Results from the Bolshoi Simulation, ApJ 740 (2011) 102, [arXiv: 1002.3660].

[40] F. Prada, A. A. Klypin, A. J. Cuesta, J. E. Betancort-Rijo, and J. Primack, Halo concentrations in the standard LCDM cosmology, (2011) [arXiv:1104.5130].

[41] M. Meneghetti and E. Rasia, Reconciling extremely different concentration-mass relations, submitted to MNRAS (2013), [arXiv:1303.6158]. 
[42] J. F. Navarro, C. S. Frenk, and S. D. M. White, The Structure of Cold Dark Matter Halos, ApJ 462 (1996) 563, [astro-ph/9508025].

[43] K. Dolag, M. Bartelmann, F. Perrotta, C. Baccigalupi, L. Moscardini, M. Meneghetti, and G. Tormen, Numerical study of halo concentrations in dark-energy cosmologies, A\&A 416 (2004) 853-864, [astro-ph/0309771].

[44] D. H. Zhao, Y. P. Jing, H. J. Mo, and G. Börner, Accurate Universal Models for the Mass Accretion Histories and Concentrations of Dark Matter Halos, ApJ 707 (2009) 354-369, [arXiv: 0811.0828].

[45] Y. P. Jing, The Density Profile of Equilibrium and Nonequilibrium Dark Matter Halos, ApJ 535 (2000) 30-36, [astro-ph/9901340].

[46] J. C. Muñoz-Cuartas, A. V. Macciò, S. Gottlöber, and A. A. Dutton, The redshift evolution of $\Lambda$ cold dark matter halo parameters: concentration, spin and shape, MNRAS 411 (2011) 584-594, [arXiv: 1007.0438].

[47] K. Rines and A. Diaferio, CIRS: Cluster Infall Regions in the Sloan Digital Sky Survey. I. Infall Patterns and Mass Profiles, AJ 132 (2006) 1275-1297, [astro-ph/0602032].

[48] R. Wojtak and E. L. Lokas, Mass profiles and galaxy orbits in nearby galaxy clusters from the analysis of the projected phase space, MNRAS 408 (2010) 2442-2456, [arXiv:1004.3771].

[49] D. A. Buote, F. Gastaldello, P. J. Humphrey, L. Zappacosta, J. S. Bullock, F. Brighenti, and W. G. Mathews, The X-Ray Concentration-Virial Mass Relation, ApJ 664 (2007) 123-134, [astro-ph/0610135].

[50] R. W. Schmidt and S. W. Allen, The dark matter haloes of massive, relaxed galaxy clusters observed with Chandra, MNRAS 379 (2007) 209-221, [astro-ph/0610038].

[51] D. E. Johnston, E. S. Sheldon, A. Tasitsiomi, J. A. Frieman, R. H. Wechsler, and T. A. McKay, Cross-Correlation Lensing: Determining Galaxy and Cluster Mass Profiles from Statistical Weak-Lensing Measurements, ApJ 656 (2007) 27-41, [astro-ph/0507467].

[52] T. Broadhurst, K. Umetsu, E. Medezinski, M. Oguri, and Y. Rephaeli, Comparison of Cluster Lensing Profiles with $\Lambda$ CDM Predictions, ApJ 685 (2008) L9-L12, [arXiv:0805.2617].

[53] A. Vikhlinin, A. V. Kravtsov, R. A. Burenin, H. Ebeling, W. R. Forman, A. Hornstrup, C. Jones, S. S. Murray, D. Nagai, H. Quintana, and A. Voevodkin, Chandra Cluster Cosmology Project III: Cosmological Parameter Constraints, ApJ 692 (2009) 1060-1074, [arXiv:0812.2720].

[54] S. Ettori, F. Gastaldello, A. Leccardi, S. Molendi, M. Rossetti, D. Buote, and M. Meneghetti, Mass profiles and $c-M_{D M}$ relation in X-ray luminous galaxy clusters (Corrigendum), A\&A 526 (2011) C1, [arXiv: 1009.3266].

[55] M. Oguri, M. Takada, K. Umetsu, and T. Broadhurst, Can the Steep Mass Profile of A1689 Be Explained by a Triaxial Dark Halo?, ApJ 632 (2005) 841-846, [astro-ph/0505452].

[56] M. Sereno and K. Umetsu, Weak- and strong-lensing analyses of the triaxial matter distribution of Abell 1689, MNRAS 416 (2011) 3187-3200, [arXiv:1105.4994].

[57] C. Giocoli, M. Meneghetti, S. Ettori, L. Moscardini, Cosmology in 2D: the concentration-mass relation for galaxy clusters, MNRAS 426 (2011) 1558-1573, [arXiv: 1205.2375].

[58] V. L. Corless and L. J. King, Cosmology with the cluster mass function: mass estimators and shape systematics in large weak lensing surveys, MNRAS 396 (2009) 315-324, [arXiv:0901.3434].

[59] L. King and V. Corless, Complex structures in galaxy cluster fields: implications for gravitational lensing mass models, MNRAS 374 (2007) L37-L41, [astro-ph/0610493]. 
[60] O. Y. Gnedin, D. Ceverino, N. Y. Gnedin, A. A. Klypin, A. V. Kravtsov, R. Levine, D. Nagai, and G. Yepes, Halo Contraction Effect in Hydrodynamic Simulations of Galaxy Formation, (2011) [arXiv: 1108.5736].

[61] M. Sereno, M. Lubini, and P. Jetzer, A multiwavelength strong lensing analysis of baryons and dark matter in the dynamically active cluster AC 114, A\&A 518 (2010) A55, [arXiv: 0904.0018].

[62] C. Fedeli, The effects of baryonic cooling on the concentration-mass relation, (2011) [arXiv: 1111.5780].

[63] A. E. Evrard, C. A. Metzler, and J. F. Navarro, Mass Estimates of X-Ray Clusters, ApJ 469 (1996) 494, [astro-ph/9510058].

[64] K. Dolag, F. Vazza, G. Brunetti, and G. Tormen, Turbulent gas motions in galaxy cluster simulations: the role of smoothed particle hydrodynamics viscosity, MNRAS 364 (2005) 753-772, [astro-ph/0507480].

[65] E. Rasia, S. Ettori, L. Moscardini, P. Mazzotta, S. Borgani, K. Dolag, G. Tormen, L. M. Cheng, and A. Diaferio, Systematics in the X-ray cluster mass estimators, MNRAS 369 (2006) 2013-2024, [astro-ph/0602434].

[66] D. Nagai, A. Vikhlinin, and A. V. Kravtsov, Testing X-Ray Measurements of Galaxy Clusters with Cosmological Simulations, ApJ 655 (2007) 98-108, [astro-ph/0609247].

[67] E. Rasia, S. Borgani, S. Ettori, P. Mazzotta, M. Meneghetti On the Discrepancy between Theoretical and X-Ray Concentration-Mass Relations for Galaxy Clusters, ApJ in press (2013), [arXiv: 1301.7476].

[68] A. Klypin, A. V. Maccio', R. Mainini, S. A. Bonometto, Halo properties in models with dynamical Dark Energy ApJ 599 (2003) 31-37, [astro-ph/0303304].

[69] A. V. Maccio', C. Quercellini, R. Mainini, L. Amendola, S. A. Bonometto, Coupled dark energy: Parameter constraints from N-body simulations, Phys. Rev. D 69 (2004) 123516, [astro-ph/0309671].

[70] M. Baldi, V. Pettorino, G. Robbers, V. Springel, Hydrodynamical N-body simulations of coupled dark energy cosmologies, MNRAS 403 (2010) 1684B, [arXiv:0812.3901].

[71] M. Baldi Time dependent couplings in the dark sector: from background evolution to nonlinear structure formation MNRAS 411 (2011) 1077, [arXiv: 1005.2188].

[72] C. De Boni, S. Ettori, k. Dolag, L. Moscardini, Hydrodynamical simulations of galaxy clusters in dark energy cosmologies - II. c-M relation, MNRAS 428 (2013) 2921-2938, [arXiv: 1205.3163].

[73] M. Schirmer, T. Erben, P. Schneider, C. Wolf, and K. Meisenheimer, GaBoDS: The Garching-Bonn Deep Survey. II. Confirmation of EIS cluster candidates by weak gravitational lensing, A\&A 420 (2004) 75-78, [astro-ph/0401203].

[74] M. Maturi, M. Meneghetti, M. Bartelmann, K. Dolag, and L. Moscardini, An optimal filter for the detection of galaxy clusters through weak lensing, A\&A 442 (2005) 851-860, [astro-ph/0412604].

[75] J. F. Hennawi and D. N. Spergel, Shear-selected Cluster Cosmology. 1: Tomography and Optimal Filtering, ApJ 624 (2005) 59-79, [astro-ph/0404349].

[76] N. Kaiser and G. Squires, Mapping the dark matter with weak gravitational lensing, ApJ 404 (1993) 441-450.

[77] N. Kaiser, G. Squires, and T. Broadhurst, A Method for Weak Lensing Observations, ApJ 449 (1995) 460, [astro-ph/9411005]. 
[78] B. Jain and L. Van Waerbeke, Statistics of Dark Matter Halos from Gravitational Lensing, ApJ 530 (2000) L1-L4, [astro-ph/9910459].

[79] P. Schneider, L. van Waerbeke, B. Jain, and G. Kruse, A new measure for cosmic shear, MNRAS 296 (1998) 873-892, [astro-ph/9708143].

[80] D. Gruen, G. M. Bernstein, T. Y. Lam, S. Seitz, Optimizing weak lensing mass estimates for cluster profile uncertainty, MNRAS 416 (2011) 1392 [arXiv:1104.2596].

[81] L. Marian, S. Hilbert, R. E. Smith, P. Schneider, and V. Desjacques, Measuring Primordial Non-gaussianity Through Weak-lensing Peak Counts, ApJ 728 (2011) L13, [arXiv:1010.5242].

[82] T. Hamana, M. Oguri, M. Shirasaki, M. Sato, Scatter and bias in weak lensing selected clusters, MNRAS 425 (2012) 2287-2298, [arXiv: 1204.6117].

[83] Z. Fan, H. Shan, J. Liu Noisy weak-lensing convergence peak statistics near clusters of galaxies and beyond, ApJ 719 (2010) 1408-1420 [arXiv: 1006.5121].

[84] Y. M. Bahe', I. G. McCarthy, L. J. King, Mock weak lensing analysis of simulated galaxy clusters: bias and scatter in mass and concentration, MNRAS 421 (2012) 1073-1088 [arXiv: 1106 . 2046].

[85] Y. P. Jing, Y. Suto, Triaxial Modeling of Halo Density Profiles with High-Resolution N-Body Simulations, ApJ 574 (2002 ) 538-553, [astro-ph/0202064].

[86] G. Hinshaw D. Larson, E. Komatsu, D. N. Spergel, C. L. Bennett, J. Dunkley, M. R. Nolta, M. Halpern, R. S. Hill, N. Odegard, L. Page, K. M. Smith, J. L. Weiland, B. Gold, N. Jarosik, A. Kogut, M. Limon, S. S. Meyer, G. S. Tucker, E. Wollack, E. L. Wright, Nine-Year Wilkinson Microwave Anisotropy Probe (WMAP) Observations: Cosmological Parameter Results, (2012), [arXiv: 1212.5226].

[87] L. Marian, R. E. Smith, G. M. Bernstein The impact of correlated projections on weak lensing cluster counts ApJ 709 (2010) 286-300, [arXiv: 0912.0261]

[88] W. H. Press and P. Schechter, Formation of Galaxies and Clusters of Galaxies by Self-Similar Gravitational Condensation, ApJ 187 (1974) 425-438.

[89] R. K. Sheth and G. Tormen, Ellipsoidal collapse and an improved model for the number and spatial distribution of dark matter haloes. MNRAS 308 (1999) 119-126, [astro-ph/9901122].

[90] A. Jenkins, C. S. Frenk, S. D. M. White, J. M. Colberg, S. Cole, A. E. Evrard, H. M. P. Couchman, N. Yoshida, The mass function of dark matter haloes, MNRAS 321 (2001) 372-384, [astro-ph/0005260].

[91] D. Reed, J. Gardner, T. Quinn, J. Stadel, M. Fardal, G. Lake, F. Governato, Evolution of the mass function of dark matter haloes MNRAS 346 (2003) 565-572 [astro-ph/0301270].

[92] M. S. Warren, K. Abazajian, D. E. Holz, L. Teodoro, Precision Determination of the Mass Function of Dark Matter Halos, ApJ 646 (2006) 881-885, [astro-ph/0506395].

[93] J. Courtin, Y. Rasera, J.-M. Alimi, P. S. Corasaniti, V. Boucher, A. FÃijzfa, Imprints of dark energy on cosmic structure formation - II. Non-universality of the halo mass function, MNRAS 410 (2011) 1911-1931, [arXiv: 1001.3425].

[94] J. M. Bardeen, J. R. Bond, N. Kaiser, A. S. Szalay, The statistics of peaks of Gaussian random fields, ApJ 304 (1986) 15-61.

[95] G. Holder, Z. Haiman, J. Mohr, Constraints on $\Omega_{m}, \Omega_{\Lambda}$, and $\sigma_{8}$ from Galaxy Cluster Redshift Distributions, ApJ 560 (2001) L111-L114, [astro-ph/0105396].

[96] Planck collaboration, Planck 2013 results. XVI. Cosmological parameters, submitted to A\&A (2013), [arXiv: 1303.5076]. 
[97] C. Heymans et al., CFHTLenS tomographic weak lensing cosmological parameter constraints: Mitigating the impact of intrinsic galaxy alignments. MNRAS 432 (2013) 2433-2453, [arXiv: 1303.1808].

[98] R. A. Burenin, A. A. Vikhlinin, Cosmological parameters constraints from galaxy cluster mass function measurements in combination with other cosmological data, AstL 38 (2012) 347-363, [arXiv:1202.2889].

[99] M. Sereno and G. Covone, The mass-concentration relation in massive galaxy clusters at redshift $\sim 1$. MNRAS 434 (2013) 878-887, [arXiv:1306.6096].

[100] A. R. Zentner, D. H. Rudd, W. Hu, Self Calibration of Tomographic Weak Lensing for the Physics of Baryons to Constrain Dark Energy. Phys. Rev. D 77 (2008) 043507, [arXiv:0709.4029].

[101] D. J. Eisenstein, W. Hu, M. Tegmark, Cosmic Complementarity: Joint Parameter Estimation from CMB Experiments and Redshift Surveys. ApJ 518 (1999) 2-23, [astro-ph/9807130].

[102] W. Hu \& B. Jain, Joint Galaxy-Lensing Observables and the Dark Energy. Phys. Rev. D 70 (2004) 043009, [astro-ph/0312395].

[103] W. Hu \& A. V. Kravtsov, Sample Variance Considerations for Cluster Surveys. ApJ 584 (2003) 207-715, [astro-ph:0203169].

[104] M. Takada \& S. Bridle, Probing dark energy with cluster counts and cosmic shear power spectra: including the full covariance. NJPh 9 (2007) 446, [arXiv:0705.0163].

[105] P. Valageas, N. Clerc, F. Pacaud, M. Pierre, Covariance matrices for halo number counts and correlation functions. A\&A 536 (2011) A95, [arXiv:1104.4015].

[106] M. Lima \& W. Hu, Self-calibration of cluster dark energy studies: Counts in cells. Phys. Rev. D 70 (2004) 043504, [astro-ph/0401559 ].

[107] M. Oguri, Self-Calibrated Cluster Counts as a Probe of Primordial Non-Gaussianity. Phys. Rev. Lett. 102 () 211301, [arXiv:0905.0920].

[108] R. H. Wechsler, A. R. Zentner, J. S. Bullock, A. V. Kravtsov, B. Allgood, The Dependence of Halo Clustering on Halo Formation History, Concentration, and Occupation. ApJ 652 (2006) 71-84, [astro-ph/0512416].

[109] Y. P. Jing, Y. Suto, H. J. Mo, The dependence of dark halo clustering on the formation epoch and the concentration parameter. ApJ 657 (2007) 664-668, [astro-ph/0610099].

[110] M. Postman, L. M. Lubin, J. E. Gunn, J. B. Oke, J. G. Hoessel, D. P. Schneider, J. A. Christensen, The Palomar Distant Clusters Survey. I. The Cluster Catalog. AJ 111 (1996) 615, [astro-ph/9511011].

[111] B. P. Koester, T. A. McKay, J. Annis, R. H. Wechsler, A. Evrard, L. Bleem, M. Becker, D. Johnston, E. Sheldon, R. Nichol, C. Miller, R. Scranton, N. Bahcall, J. Barentine, H. Brewington, J. Brinkmann, M. Harvanek, S. Kleinman, J. Krzesinski, D. Long, A. Nitta, D. Schneider, S. Sneddin, W. Voges, D. York, SDSS collaboration, A MaxBCG Catalog of 13,823 Galaxy Clusters from the Sloan Digital Sky Survey. ApJ 660 (2007) 239-255, [astro-ph/0701265].

[112] M. Milkeraitis, L. Van Waerbeke, C. Heymans, H. Hildebrandt, J. P. Dietrich, T. Erben, 3D-Matched-Filter galaxy cluster finder - I. Selection functions and CFHTLS Deep clusters. MNRAS 406 (2010) 673-688, [arXiv:0912.0739].

[113] F. Bellagamba, M. Maturi, T. Hamana, M. Meneghetti, S. Miyazaki, L. Moscardini, Optimal filtering of optical and weak lensing data to search for galaxy clusters: application to the COSMOS field. MNRAS 413 (2011) 1145-1157, [arXiv:1006.0610]. 
[114] L. Campanelli, G. L. Fogli, T. Kahniashvili, A. Marrone, B. Ratra, Galaxy cluster number count data constraints on cosmological parameters, (2011), [arXiv:1110.2310].

[115] V. Cardone, S. Camera, M. Sereno, G. Covone, R. Maoli, R. Scaramella, (2014), in preparation. 